January 6, 2005

\title{
Manual Tracking in Three Dimensions
}

\author{
Leigh A. Mrotek ${ }^{1}$, CCAM Gielen ${ }^{2}$ and Martha Flanders ${ }^{1}$ \\ ${ }^{1}$ University of Minnesota, Department of Neuroscience \\ ${ }^{2}$ Radboud University Nijmegen, Department of Medical Physics and Biophysics
}

Running head: 3D hand tracking

Correspondence:

M. Flanders

Department of Neuroscience

6-145 Jackson Hall

312 Church St. S.E.

University of Minnesota

Minneapolis MN 55455

phone: (612) 624-6601

FAX: (612) 626-5009

e-mail: fland001@umn.edu

Key words: smooth pursuit, anticipatory response, prediction, hand tracking, arm movement, two-thirds power law 


\begin{abstract}
The purpose of this study was to examine the manual tracking of a virtual target, moving in 3-dimensions. Two basic types of target paths were used: a peanut-shaped Cassini ellipse and a more spherical shape where four connected semi-circles lay in horizontal and vertical planes (“4-Plane shape”). The Cassini shapes were presented in frontal and side orientations and the 4-Plane shape was presented in three different sizes. The Cassini shapes were also folded along one of three axes, where the bends brought the target path into another plane. During the smooth sections of the target trajectory, subjects were able to track accurately, presumably due to anticipation. However, when the target passed through a bend in the shape, this caused large errors in tracking position and direction. Overall tracking performance improved during the first three seconds of the first cycle, and then stabilized for the last two seconds of the first cycle and for subsequent cycles. In the first cycle, the hand lagged target motion by about $150 \mathrm{~ms}$ on average, which decreased to $100 \mathrm{~ms}$ in the last three cycles. Tracking in depth was compared to tracking in the frontal plane. Subjects showed little modulation in depth position resulting in a flattening of the shape. They also had inferior hand/target correlations for depth position and velocity, with correlation coefficients of about $0.7-0.8$. These values were considerably better for hand tracking in the frontal plane, where correlation coefficients ranged from 0.92-0.99.
\end{abstract}




\section{Introduction}

Early studies of hand and eye tracking hinted at various reactive, predictive and anticipatory mechanisms (Poulton 1974, Yasui and Young 1975). More recent experiments show that during hand or eye tracking in one-dimension, subjects require only a few trials to begin to anticipate the speed or frequency of a target repeatedly moving at the same constant speed or oscillation frequency (Barnes and Marsden 2002, Barnes et al. 2000); this phenomenon may be related to the well-known ability of humans to follow low frequency, sinusoidal target motion with no time lag (Dallos and Jones 1963). Well-trained monkeys can also follow, with the eyes, more complex, twodimensional target motions, and they do this with zero lag for low frequencies and lags gradually approaching 100 ms for higher frequencies (Kettner et al. 1996). However, in spite of these and many other similar observations, the possible types and mechanisms of anticipation and prediction remain unclear, especially for targets that travel along curved trajectories in two or three dimensions.

When subjects move the hand in two or three dimensions, they tend to follow several “rules” including: 1) use of small speed pulses for error corrections, 2) piecewise planar hand paths due to maintenance of a particular phase relation between elbow and shoulder angles, and 3) a lawful relation between hand speed and hand path curvature (Roitman et al. 2004; Soechting and Terzuolo 1986, 1987b; Viviani et al. 1987). These rules may also be followed during the tracking of complex target trajectories, and these characteristics may change over the course of several repetitions, as the shape and timing of the target path and trajectory become familiar. 
Little work has been done on manual tracking in three dimensions (3D), but there are several reasons to hypothesize that tracking performance in depth is relatively poor. Oculomotor tracking of targets in 3D is much better for azimuth and elevation, than for depth (vergence) (Gielen et al. 2004). Since gaze affects the accuracy of pointing (see e.g., Bock 1986; Henriques et al. 1998; Medendorp and Crawford 2002), errors in ocular tracking will affect the accuracy of hand tracking. This is in agreement with the observation that errors for pointing to remembered targets in three dimensions are greater for the depth dimension than for the other two dimensions (Adamovich et al. 1998; Soechting and Flanders 1989a,b; Admiraal et al. 2003).

In the present study, we examined three basic questions. First, we determined whether or not subjects gradually improved hand tracking performance while tracking a target that moved four times in succession, along the perimeter of a complex 3D shape. We also examined the ability of subjects to abruptly change the plane of hand motion, by testing tracking performance when the target abruptly changed direction. The final aspect of our study was a comparison of hand tracking performance across the three dimensions of the target motion; i.e., we compared tracking in depth to tracking in the horizontal and vertical dimensions.

\section{Methods}

The results of our experiment will be reported in a set of two papers: this paper (Mrotek et al. submitted) and a companion paper (Flanders et al. submitted). In each recording session, a human subject first tracked a target that moved along the perimeter of a 3D shape ("tracking condition"). The target was a sphere with a diameter of $2.5 \mathrm{~cm}$, 
that moved along an unseen path. The subject then traced the same target path, now fully visible ("tracing condition”). Finally, the subject drew the shape, in the same spatial location, from memory (“drawing condition”). This first paper (Mrotek et al. submitted) examines the manual tracking performance in the tracking condition, whereas the second paper (Flanders et al. submitted) compares several other aspects of the arm movements (e.g., planarity and speed/curvature relations) across all three conditions (tracking, tracing and drawing).

\section{Target Shapes}

We used two basic types of target path: a peanut-shaped Cassini ellipse (“Cassini shape,” Fig. 1A and Fig. 2) and a more spherical shape where four connected semi-circles lay in horizontal and vertical planes (“4-Plane shape,” Fig. 1B). The Cassini shapes were modified by showing them from front and side orientations, and they were also folded along one of three axes (Table 1). The 4-Plane shapes were shown in three different sizes (Table 1).

\section{Cassini ellipse shapes}

Eight of the fourteen Cassini shapes are shown in Fig. 2. All of the shapes are rendered from the subject's perspective; the subject's eyes were aligned with the center of the shape. The shapes were comprised of planar curved segments, and to make the target motion as biological as possible, within each plane we programmed the target speed to approximately obey the 2/3 power law (Lacquaniti et al. 1983). Thus, speed changed throughout the Cassini shapes, but the average speed was $26 \mathrm{~cm} / \mathrm{s}$.

In Fig. 2A and Fig. 2B, we show the basic shape without bends, in frontal and side orientations, respectively. When viewed from the subject's perspective or from 
above, the target always traveled clockwise around the unseen path. The word "indent" will be used to refer to the impressions between the two main lobes of the shape; they occurred at times $1250 \mathrm{~ms}$ and $3750 \mathrm{~ms}$ in each $5000 \mathrm{~ms}$ cycle. The equations used to make the basic (Front No Bend) Cassini shape were:

$$
\begin{aligned}
& X_{i=1: 300}=R *(1+A * \cos (2 i)) * \cos (i), \\
& Z_{i=1: 300}=1.5 * R *(1+A * \cos (2 i)) * \sin (i),
\end{aligned}
$$

where $R=32 \mathrm{~cm}$ and $A=0.5$. We used a right-handed coordinate system with the $Z$-axis in the upward direction, the $X$-axis in the frontal plane, horizontal direction, and the $Y$-axis in depth. The width of the shape was $32 \mathrm{~cm}$ and the long axis was $48 \mathrm{~cm}$.

To give them depth, the Cassini shapes were then rotated $45^{\circ}$ around the horizontal axis through the center of the shape. For the frontal orientation (Fig. 2A), the bottom of the shape was closer to the subject (thus the bottom appears larger in the 3D rendering). For the side orientation (Fig. 2B), the shape was also rotated $80^{\circ}$ around the vertical axis. Another difference between the front and side orientations was that the starting position was at the top for the front orientation and at the bottom for the side orientation. In Fig. 2, starting position is depicted as a discontinuity.

Figure 1A and Table 1 list the axes along which the Cassini shapes were folded. The word "bend" will be used to refer to a perimeter location where the shape was folded. The bends were either $30^{\circ}$ or $60^{\circ}$ (see Table 1), but only the $60^{\circ}$ bends are shown in Fig. 2. Figures 2C and 2D show the shape bent along the long axis for the frontal and side orientations, respectively. Figures $2 \mathrm{E}$ and $2 \mathrm{~F}$ show the shape bent along the short axis, and Figs. 2G and 2H show the shape bent along the oblique axis. 


\section{4-Plane shapes}

The 4-Plane shapes were made from four semi-circles, each placed in a plane perpendicular to the plane of the previous semi-circle (Fig. 1B). Two semi-circles were in horizontal planes and the openings faced away from the subjects; two were in parasagittal planes and the openings faced towards the subject. Each semi-circle for the small 4-Plane shape had a radius of $8 \mathrm{~cm}$; therefore the shape was $16 \mathrm{~cm}$ in the frontal plane and $16 \mathrm{~cm}$ in depth. The medium 4-Plane shape had radii of $14 \mathrm{~cm}$, and the large 4-Plane shape had radii of $20 \mathrm{~cm}$. For the small shape, the target moved at a constant speed of $11.42 \mathrm{~cm} / \mathrm{s}$, for the medium shape the speed was $19.99 \mathrm{~cm} / \mathrm{s}$, and for the large shape the speed was $28.56 \mathrm{~cm} / \mathrm{s}$.

\section{Procedures}

All experimental procedures were in accordance with the human subjects regulations of Radboud University Nijmegen, where the recording sessions took place. Six subjects volunteered for the experiment (4 females and 2 males; 4 right handed and 2

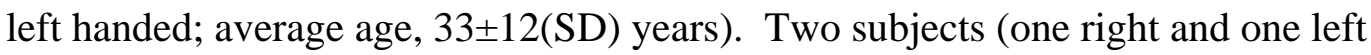
handed) repeated the experiment and for these subjects, corresponding data (such as error or lag) from the two sessions were averaged. Subjects were presented with the shapes in a pseudorandom order; each of the 17 shapes was presented once (i.e., 7 Cassini shapes in frontal view, 7 Cassini shapes in side view, and three 4-Plane shapes). For every shape, subjects were first asked to track a single moving target, and then after short rests of about $30 \mathrm{~s}$, they traced, and then drew from memory. For the tracking trials, the target continued to move around the shape four times. Each complete revolution around the shape will be termed a “cycle.” 


\section{Measurement system}

Infra-red light-emitting diode markers were placed on the right shoulder, elbow, and wrist, and on the tip of a pen-like object held in the right hand. Subjects were asked to track, trace or draw with the tip of the pen, and were required to refrain from bending the wrist. The 3D locations of the markers were recorded with an Optotrak 3020 system (Northern Digital Inc.) at $100 \mathrm{~Hz}$ and with a precision of better than $0.15 \mathrm{~mm}$ in all dimensions. For the analyses presented in this paper, we only examined the movement of the marker on the pen tip (the "hand" marker).

\section{Target projection system}

Targets were produced using a virtual reality system. The subject sat with the head at a distance of $90 \mathrm{~cm}$ from a vertical screen. Two projections of the ball in red and green (as viewed from the right and left eye, respectively) were projected onto the screen with an LCD projector (Philips 4750). Custom software was used to calculate the desired locations and sizes of the balls (when viewed through red and green 3D glasses, for the right and left eyes, respectively). This binocular presentation gave the impression of a sphere moving through space.

The perceived depth position of the sphere also depended on the distance between the eyes, and since subjects had varying distance between their pupils (range 5.4$6.25 \mathrm{~cm}$ ), this depth position was slightly different for the various subjects. Therefore, in post hoc analysis, we calibrated the target trajectory based on an estimate of where each subject actually saw the target. To do this we used the subject's reproduction of the shape in the tracing condition. First, we used regression analysis to find the best parameters to center and scale the target path to the subject's tracing path for each shape 
individually. Then, we again used regression analysis to scale all of these transformed target paths to the subject's general gain and bias. The calibrated shape was usually nearly identical to the programmed target path. For example, the target shapes shown in Fig. 3 (thick dashed lines) are the result of the calibration procedure.

Data analysis

\section{Cycles and sections}

For each shape, the target moved in four consecutive cycles. For the Cassini shapes, each target cycle lasted $5 \mathrm{~s}$; for the 4-Plane shapes, each target cycle lasted $6.7 \mathrm{~s}$. During analysis, each cycle of each Cassini shape was separated into sub-cycles, which were defined by the six possible bends and indents (see Fig. 1A).

\section{Position and velocity}

Data were collected in a three-dimensional coordinate system and saved for offline analysis. The right-handed coordinate system was defined such that the frontal, horizontal dimension was the $X$-axis (positive to the right of the subject), the depth dimension was the $Y$-axis (positive forward from the subject), and the vertical dimension was the $Z$-axis (positive upward).

The position of the target and hand was graphed over time for each dimension. For example, Fig. 4 (left column, thick dashed line) shows the $X$-, $Y$ - and $Z$-coordinates of the target trajectory for one Cassini shape (Side $60^{\circ}$ Long). Note the magnified scale for the $Y$ - (depth) dimension in Fig. 4C. The position data were differentiated to compute velocity. The velocity over time for the Side $60^{\circ}$ Long shape is depicted in the right column of Fig. 4 (thick, dashed line). 
In each panel in Fig. 4, there are four vertical lines. As indicated in Fig. 4E, the double vertical lines denote the times of the midpoint of each indent (1250 ms and $3750 \mathrm{~ms}$ ) and the single vertical lines denote the times when an abrupt bend occurred. For the Side $60^{\circ}$ Long shape, the bends were located at the middle of the shape (2500ms) and at end of each cycle (0 and $5000 \mathrm{~ms})$.

\section{$\underline{\text { Direction }}$}

The direction of the target and hand motion was calculated in the frontal plane and in the sagittal plane by using the velocity from the dimensions that make up each plane:

$$
\begin{aligned}
& \Theta_{F P}=\tan ^{-1} \frac{V_{z}}{V_{x}} ; \\
& \Theta_{S P}=\tan ^{-1} \frac{V_{z}}{V_{y}} .
\end{aligned}
$$

$\Theta_{F P}$ represents direction in the frontal plane and $\Theta_{S P}$ represents direction in the sagittal plane (see Fig. 5 for an example).

\section{Distance, speed and direction error}

For each point in time, we calculated the distance between the 3D target and hand positions (distance error, $\left.E_{d i s}\right)$ :

$$
E_{d i s}(t)=\sqrt{\left(P_{X T}(t)-P_{X H}(t)\right)^{2}+\left(P_{Y T}(t)-P_{Y H}(t)\right)^{2}+\left(P_{Z T}(t)-P_{Z H}(t)\right)^{2}} .
$$

The subscript $T$ indicates target position and the subscript $H$ indicates hand position.

We used two methods to calculate the difference between the target and hand speed. First we found the signed speed error $\left(E_{ \pm s p d}\right)$ by subtracting the hand speed from the target speed: 


$$
E_{ \pm s p d}(t)=\sqrt{\left(V_{X T}(t)\right)^{2}+\left(V_{Y T}(t)\right)^{2}+\left(V_{Z T}(t)\right)^{2}}-\sqrt{\left(V_{X H}(t)\right)^{2}+\left(V_{Y H}(t)\right)^{2}+\left(V_{Z H}(t)\right)^{2}}
$$

We also computed the unsigned speed error $\left(E_{s p d}\right)$ in a manner similar to the calculation of the distance error:

$$
E_{s p d}(t)=\sqrt{\left(V_{X T}(t)-V_{X H}(t)\right)^{2}+\left(V_{Y T}(t)-V_{Y H}(t)\right)^{2}+\left(V_{Z T}(t)-V_{Z H}(t)\right)^{2}} .
$$

We calculated the direction error in the two planes by finding the absolute difference between the target and hand direction:

$$
E_{\text {dir }}=\left|\operatorname{Dir}_{T}-\operatorname{Dir}_{H}\right|
$$

The direction error was calculated in the frontal plane and the sagittal plane separately.

\section{Position, velocity and direction lag/lead}

We tested whether the subjects were responding to the target motion or anticipating it by calculating a lag/lead measure for position, velocity and direction. Lag is given a negative value and lead is given a positive value. Cross-correlation analysis was used to find the overall time lag/lead for each cycle. All times from -350 to $+350 \mathrm{~ms}$ were tested in $10 \mathrm{~ms}$ intervals to determine the lag and the correlation coefficient. In order to test for differences between various correlation coefficients, we converted them to Z-scores.

If the cross correlation analysis did not reveal a significant peak within the $+/-350$ ms time interval, the cycle was excluded from statistical analysis. Data representing the depth dimension or the sagittal plane had many more cycles excluded than did data representing the horizontal or vertical dimensions, or the frontal plane. Of the 408 total cycles, those excluded were: 79 for depth position lag, zero for horizontal position lag, one for vertical position lag, 29 for depth velocity lag, one for horizontal velocity lag, and 
one for vertical velocity lag. The sagittal plane direction lag had 12 cycles excluded, whereas the frontal plane direction lag had only 1 cycle excluded.

\section{Instantaneous lag}

In addition to using cross-correlation analysis to find the overall lag for the entire cycle, we also wanted to find the lag for each hand position, within each cycle (see Fig. 8). To do this, for each instantaneous hand position, we calculated the distance to a range of possible positions of the target. We included all target positions from one second before the current time until the current time. Thus, we generated a vector of distance error values:

$$
\operatorname{Error}_{i}(t)=\sqrt{\left(X_{H(t)}-X_{T(i)}\right)^{2}+\left(Y_{H(t)}-Y_{T(i)}\right)^{2}+\left(Z_{H(t)}-Z_{T(i)}\right)^{2}} .
$$

where the index $i$ is the sample interval, which ranges from the current time at this hand position ( $t$ ) to the time $1000 \mathrm{~ms}$ earlier $(t-1000 \mathrm{~ms})$. Thus, the subscript $H(t)$ indicates the hand position at the current time and the subscript $T(i)$ indicates all of the target positions from the current time to one second earlier. The result of Eq. 9 was a vector of error values for each hand position. We then searched for the minimum Errori $(t)$ value and found the time difference between the time when the target was the closest $\left(\operatorname{Time}\left(\min \left(\operatorname{Error}_{(}(t)\right)\right)\right)$ and the current time $t$ :

$$
\operatorname{Lag}=\operatorname{Time}\left(\min \left(\operatorname{Error}_{i}(t)\right)\right)-t .
$$

This time difference will be called the instantaneous lag ( $\mathrm{Lag})$. 


\section{Results}

\section{Tracking position in three dimensions}

The path of the hand marker approximated the calibrated target path. Fig. 3 displays position data for four shapes, tracked by one subject. The thick dashed lines represent the path of the target, the thick solid line shows the hand tracking for the first cycle, and the thinner lines represent the second, third and fourth cycles. The first cycle had greater error. For example, in Fig. 3A the thick line starts out (arrow) farther away from the target line. This is also seen for the top semi-circle in the Medium 4-Plane shape (Fig. 3B) and the first quarter of the Side $60^{\circ}$ Long shape (Fig. 3D). The tracking for the next three cycles was fairly consistent. Across all conditions and all subjects, the tracking also appeared to be better in the frontal plane than in depth.

In the left column of Fig. 4, we show, for each dimension, the position of target and hand motion for one subject, for the Side $60^{\circ}$ Long shape. The results displayed here are typical of all subjects. The dashed line represents the target motion, the thick solid line represents the hand motion during the first cycle, and the thinner lines represent the hand motion for the remaining cycles. As explained under Methods, the vertical double lines represent the times when the target was at the center of an indent and the single vertical lines represent the times when an abrupt bend occurred.

The subject was very accurate in tracking the target in the frontal plane, in both the horizontal and the vertical dimensions (Fig. 4A and Fig. 4E). There was little position error, and the subject was fairly consistent across cycles. For the vertical dimension, the most error appeared to occur in the first $500 \mathrm{~ms}$ of each cycle, when the target was traveling mostly upward (Fig. 4E). For the horizontal dimension, the most 
error occurred at the middle of each cycle; this is when the target motion had an abrupt bend and reversed horizontal direction (2500 ms; Fig. 4A). The subject also typically lagged behind the target, as evidenced by the hand data being shifted to the right relative to the target data.

The target position was not tracked as well in depth, and thus there were large depth position errors across the entire shape. For example, in Fig. 4C, the hand data show little modulation. During tracking, subjects typically made the shape flatter than the traced target path (which was used for calibration). To quantify this, we calculated the ratio between the range of hand position and the range of calibrated target position. We averaged the ranges of the four cycles to get one value for each trial, and then averaged across the 17 shapes and 6 subjects $(\mathrm{n}=102)$. The result was $0.82(+/-0.19$ SD) for depth, compared to 0.98 (+/-0.08 SD) and 1.04 (+/-0.11 SD) for the horizontal and the vertical dimensions, respectively. ANOVA with Scheffé's posthoc test showed that the value for the $Y$-dimension was significantly lower than for the $X$-and $Z$-dimensions, and also that the value for $X$ was less than for $Z(\mathrm{~F}(2,303)=72.00$, $\mathrm{p}<0.001)$.

\section{Tracking velocity in three dimensions}

Velocity data for each dimension (for the same trial) are shown in the right column of Fig. 4. Target velocity was tracked very well, especially in the horizontal and vertical dimensions (Fig. 4B and Fig. 4F). Furthermore, the subject’s velocity patterns were consistent across the cycles for each dimension, especially for cycles 2-4 (thin lines). However, the hand velocity lagged behind the target velocity, most obviously just after the target had a large change in velocity. For example, in the horizontal dimension, 
between 1250 and $2500 \mathrm{~ms}$, the target velocity changed rapidly. The hand velocity followed this change with a slight lag.

In contrast to depth position, where there was little similarity between traces representing the target and the hand (Fig. 4C), the hand's depth velocity clearly followed some of the large, smooth fluctuations in the target's depth velocity (Fig. 4D). This suggests that subjects may perform poorly for slow changes in target depth position, but may track the smooth, fast changes quite well. This would indicate that the tracking in depth is less responsive to the lowest frequencies in the target motion.

The influence of the target bends (the highest frequency in the target motion) was quite apparent in the hand velocity traces. For the bend at $2500 \mathrm{~ms}$ (single vertical line in the middle of each graph), the target suddenly traveled in a direction that would not have been predicted from a smooth continuation of the target's trajectory prior to the bend. The subject responded with an overshoot in horizontal velocity (Fig. 4B) and responded substantially later in depth velocity (Fig. 4D). These results illustrate that abrupt bends in the target trajectory could be quite disruptive to hand tracking.

\section{Tracking direction in the frontal and sagittal planes}

We found that the pattern of changes in hand direction matched the pattern of changes in target direction with a lag and some error (Fig. 5). The indents were not noticeably disruptive in position and velocity (see Fig. 4), but they produced many errors in direction (near the double lines in Fig. 5). During the indents, subjects often made small loops, with direction changing by $360^{\circ}$. Because the direction measure is circular, on the graphs in Fig. 5, a full rotation would appear as a smooth change in direction with a single, abrupt reversal at some point. An example of this is seen in the plot of sagittal 
plane direction (Fig. 5B) just prior to the midpoint of the first indent (approximately $1050 \mathrm{~ms}$ ) for the final two cycles (thin lines). Due to the point of view of the 3D graphs in Fig. 3D, it is difficult to determine whether the subject made a loop in the sagittal plane. However, we examined these cycles in detail and found that the subject did make a small loop on each of the final two cycles. Interestingly, a similar abrupt change in direction is not seen in the frontal plane direction because, while the hand made a change of $360^{\circ}$ in direction from the sagittal view, it maintained correct motion in the frontal plane. Thus the 3D shape of the hand motion was a spiral.

In accord with the 2/3 power law (Lacquaniti et al. 1983) and the time course of the target motion, the hand slowed down during the tight curvature of the indent in the target path. Since direction was calculated using velocity values, if the $X$ - or $Y$ component of velocity became zero, the directions in the fronto-parallel plane (Eq. 3) and the sagittal plane (Eq. 4) were undefined and the direction changed spuriously (as in the first cycle at the end of the second indent). Thus, the direction sometimes appears to change rapidly just because the subject slowed down.

The subject required a great deal of time to respond to the bends (single vertical lines Fig. 5). After the bend at $2500 \mathrm{~ms}$ the target was traveling in a direction that was not predictable as a continuation of the previous motion. In both planes, the subject made a quick correction to align the hand motion with the direction of target motion. The correction was made in the frontal plane slightly sooner than the correction in the sagittal plane. 


\section{Distance and speed error during tracking}

Average distance error for all subjects and Cassini shapes is shown in Fig. 6A for the first cycle (red line) and for the combination of all the other cycles (blue line). The vertical line hatching around each trace represents the 95\% confidence interval. As in previous Figures, the double vertical lines represent the time of the midpoint of indents and the single vertical lines show the times of all possible bends. (The long axis bends occurred at 2500 and $5000 \mathrm{~ms}$, the oblique axis bends occurred at 2000 and $4500 \mathrm{~ms}$, and the short axis bends occurred at the midpoints of the indents.)

The pattern of distance errors was similar across the cycles. There was a large error at the beginning of the cycle, then a reduction in that error beginning around 400 ms. Furthermore, after each of the indents and bends there was a small increase in distance error. Between $1250 \mathrm{~ms}$ (first set of double lines) and $3000 \mathrm{~ms}$, the average distance error rose due to the indent and because of the increased occurrence of bends. The distance error decreased during the later half of the cycle, perhaps because subjects had a better estimate of the target shape after completing the first half. The error during the first cycle (red line) was considerably larger than during the other cycles (blue line) for the first $750 \mathrm{~ms}$, and appeared slightly larger than the other cycles until approximately $3000 \mathrm{~ms}$

For all cycles, the unsigned speed error was large at the beginning of the cycle, and after each possible bend (Fig. 6B). The signed speed error indicates that the subjects tended to slow down (negative error) after an indent or bend (Fig. 6C). Unsigned speed error decreased during times when the target motion was smooth (no bends or indents). 
During these times the signed speed error was positive, indicating that the subject used the smooth portions of the target motion to catch-up.

The first cycle was somewhat different from the others in that the unsigned speed error was much larger for the first $750 \mathrm{~ms}$ while the subject was responding to the onset of the target motion and catching up to the target (Fig. 6B). When the subjects did respond, they had to move faster to catch-up to the target. They were moving slower than the target for the first $375 \mathrm{~ms}$, and then faster than the target from $375 \mathrm{~ms}$ to $1350 \mathrm{~ms}$ (Fig. 6C). The unsigned error remained elevated compared to the other cycles until approximately 3500 ms.

Distance, speed and direction error and lag across the cycles

In our measures of position, velocity and direction, it appears that the errors were largest in the first cycle. Thus, we tested for differences in the magnitude of errors and the time lags across the four cycles. The results of these analyses show that indeed there was more error and a greater lag during the first cycle (Fig. 7).

The first cycle had significantly more error than all the other cycles in over half the measures (open symbols in Fig. 7, see Table 2 for details). Furthermore, the trend was evident in all measures except for the Frontal Plane (FP) Direction Error (Fig. 7E). For some of the individual measures, not all subsequent cycles were significantly different from the first, but no data were contrary to the trend (see post hoc results in Table 2). Cycles two through four were always the same in every measure. Thus, these results indicate that much improvement was made during the first cycle and that little improvement was made thereafter. 
In contrast to the other lag values (Fig. 7B, D and F), the depth position ( $Y$ ) lead/lag shows a slight lead for the final three cycles. As discussed below (and shown in Table 4), cross-correlation values in depth, especially for position, were much lower than the values for the horizontal and vertical dimensions. This was due to the subjects' lack of overall depth modulation. The subjects that had the least modulation in depth were those most likely to show position lead values. For example, the cross-correlation on the position data in Fig. 4C resulted in an average lead value of $24 \mathrm{~ms}$; however, the depth velocity data for this same trial (Fig. 4D) resulted in a lag of $-12 \mathrm{~ms}$. As shown in Fig. 7D, velocity lags for all three dimensions showed steady-state lags around $-100 \mathrm{~ms}$ for cycles two through four. For most parameters lag was around $-150 \mathrm{~ms}$ for the first cycle and $-100 \mathrm{~ms}$ for cycles two through four.

Instantaneous lag during each cycle

It was possible that in the first cycle, our error and lag measures were biased by the unavoidably large errors during the reaction time; this might largely account for the result shown in Fig. 7. Thus we created a measure of instantaneous lag (shown schematically in Fig. 8A), which allowed us to document fluctuations in lag and to determine the time course of the improvement in performance within the first cycle.

The instantaneous lag for several shapes is presented in Fig. 8B-E (the same shapes as in Fig. 3). As in the previous Figures, data from the first cycle are shown with a thick line and data from the other cycles are shown with the thinner lines. Inevitably, due to the reaction time, the first part of the first cycle had more lag than the other cycles. However, the first cycle also had more lag than subsequent cycles until approximately $3000 \mathrm{~ms}$. 
When we examined the average of all the Cassini shapes, we found that the first cycle had more lag than the other cycles for about three of the five seconds (Fig. 8F). Due the visual reaction time and the time needed to catch up to the target, for approximately the first $1000 \mathrm{~ms}$ of the first cycle (red line), there was much more lag than for the other cycles (blue line). Around the time when the target slowed down for the indent (1000-1500 ms), the lags were similar across all four cycles, but from 1690$3150 \mathrm{~ms}$, the lag in the first cycle was again significantly greater than the lag in the other cycles. Thus for the majority of the first three seconds of the first cycle, the subjects had more lag than in the other cycles. These results show that the main effect of increased lag during the first cycle is not simply due to the reaction time for starting the trial; it continues for about three seconds.

Tracking error comparisons across the sections of the Cassini shapes

We examined the influence of the bends on performance by comparing the error and lag measures across comparable sub-cycles of the different shapes. If the bend caused a disruption in tracking, a sub-cycle after a bend would have more error than the comparable sub-cycle of a shape where there was no bend. The results of the analysis are detailed in Table 3 and drawn schematically in Fig. 9, where the entire Cassini shape is redrawn to represent each error measure. For example, the distance error is represented by the inside shape and the instantaneous lag measure is the outermost shape. Each set of shapes corresponds to a specific bend type: Long (A), Short (B), and Oblique (C). The shaded areas indicate the sections of the shapes in which our hypothesis would predict more error if the bends were disruptive. The thick dashed lines indicate the sections 
where the error was significantly larger (see Table 3 ). In accord with our prediction, the highlighted sections for each bend type contain the most dashed lines.

Except in one case, the sub-cycles in the shape without bends did not have more error than the sub-cycles following bends (Table 3; frontal plane (FP) direction error in Section 3). For shapes that had a long axis bend (Fig. 9A), there was more speed and direction error and the lag was longer at the beginning of the shape (Section 1) and in the sub-cycle after the center of the shape (Section 4; section numbers are defined in Fig. 1A). For shapes with a short axis bend (Fig. 9B), there tended to be more error and longer lags in the sections just after the bends (Sections 2 and 5), but not in as many measures as for the other bend types. This may be due to the fact that short axis bends occurred in conjunction with indents, which also caused errors in every shape type. The oblique bends caused the most error (Fig. 9C). Not only was the error larger just after the oblique bends (Sections 3 and 6) but also in other sections, suggesting that the irregularity of this shape may have degraded overall performance.

\section{Tracking error comparisons across the three dimensions}

From the position and velocity graphs in Fig. 4, and the summary data in Fig. 7, it appears that the subjects did not track as well in depth as they did in the horizontal and vertical dimensions. To further quantify the differences between tracking in each dimension, we used a measure of the strength of the relationship between the target and hand motion obtained when evaluating the time lag/lead for each dimension or plane.

As shown in Tables 4 and 5, for both position and velocity, during all the shapes, the relationship between target motion and hand motion was very high in the $X$ - and $Z$ dimensions, with correlation coefficients ranging from 0.996 to 0.922 . In contrast, 
correlations between the target and hand motion in the $Y$-dimension (depth) was significantly lower than for both the $X$ - and $Z$-dimensions, ranging from 0.849-0.628. Furthermore, as mentioned under Methods, compared with the $X$ - and $Z$-dimensions, a much greater percentage of depth position and velocity data were eliminated from this analysis due to insignificant correlations (19\% for depth position and $7 \%$ for depth velocity). Overall, these results indicate that the tracking in depth is not a good as tracking in the horizontal $(X)$ and vertical $(Z)$ dimensions for the Cassini shapes (Table 4) as well as the 4-Plane shapes (Table 5).

\section{Discussion}

We examined three main questions regarding subjects' ability to track, with the hand, targets moving in 3D. First, we examined the improvement during the course of tracking multiple cycles around a given shape: tracking improved during the first three seconds of the first cycle, and then stabilized for the last two seconds and across cycles two through four. We also examined the errors resulting from tight path curvatures (indents) and abrupt changes in target trajectory (bends); we documented the marked fluctuations in tracking performance due to these features (Figs. 4-6 and Figs. 8-9). Finally, we compared tracking in depth to tracking in the frontal plane. As expected, tracking in depth differed both qualitatively and quantitatively: subjects showed little modulation in depth position and inferior target/hand correlations for both position and velocity. 


\section{Anticipatory tracking and internal models}

We were somewhat surprised to find that tracking improved so dramatically after three seconds (Fig. 6 and Fig. 8). In previous studies, steady state performance in hand or eye tracking was reached after one or two brief trials (Barnes and Marsden 2002, Barnes et al. 2000). It could be that improvements in the gain and lag of tracking simply require a certain amount of practice. However, in our study, we also noticed that for the Cassini shapes, three seconds corresponded to movement of the hand $3 / 5$ of the distance around the shape. This put the hand beyond most of the bends and on its way into the second indent (see Fig. 8F). Since the shapes were symmetrical, it seems possible that, at the 3/5 point, the tracking system may have begun to draw upon an estimate of the remaining target path.

Visual reaction time during hand-tracking is known to be about 100 ms (Smith and Bowen 1980; Zelaznik et al. 1983; Prablanc and Martin 1992) and therefore, lags better than -100 ms suggest some form of anticipation. In addition to the dramatic overall improvement after three seconds, subjects exhibited fluctuations between zero and $-300 \mathrm{~ms}$ lag, depending on specific features of the target trajectory. The full-cycle velocity lags calculated using cross-correlation (Fig. 7D), represent a compromise across epochs of the tracking performance with various lags. Thus the improvement from -150 ms overall lag in the first cycle to a value of $-100 \mathrm{~ms}$ in cycles two through four indicates that beyond the first three seconds, numerous tracking epochs had lags substantially less than -100 ms (see Fig. 8).

The improvement in hand tracking after three seconds may be analogous to the gradual improvement in reaching movements as subjects form an "internal model" of the 
physical properties of a novel environment (e.g., Krakauer et al. 1999). The concept of the internal model has been very valuable to the field of motor control (Wolpert et al. 1995; Desmurget and Grafton 2000). The internal model is a mapping between the desired movement and the motor commands necessary to produce that movement. The proper mapping depends critically upon the physical properties of the object to be moved, and the brain is thought to store anticipated properties and to use experienced properties to update this stored representation. Since hand tracking is essentially a process of interacting with a moving target, learning to anticipate features of the trajectory may be akin to internal model learning. An internal representation of the anticipated target trajectory would allow the system to predict and evaluate the expected consequence (i.e. the desired error reduction) of each incremental tracking movement. Frequency response and predictive corrections

In the present set of experiments, target trajectories were designed to be symmetric and therefore relatively easy to remember for reproduction (in the memory condition reported in the companion paper, Flanders et al. submitted). The shapes were also broken into planar segments in accord with the hypothesis that it is easy to move the hand in a plane but difficult to change planes (Soechting et al. 1986; Soechting and Terzuolo 1987a, b). For the tracking trials, we also chose to program the target to move in good approximation to the 2/3 power law (Lacquaniti et al. 1983, see Flanders et al. submitted), and this resulted in trajectories with a range of speeds and curvatures, interspersed between sharp bends. As expected, subjects tracked better during the smooth segments (Viviani et al. 1987). 
Some of the fluctuations in tracking performance within each cycle may essentially represent the "frequency response" of the tracking system, in that certain accelerations are beyond the capabilities of the system. As mentioned in the Introduction, Kettner and colleagues (1996) evaluated the frequency response of 2D smooth pursuit eye movements and found a progressive increase in lag for higher frequency target motions. In our study, the abrupt bends may be regarded as high frequencies in the target trajectory.

As discussed above, for the lower frequencies, the improved lags may represent some form of anticipation or prediction. In principle, signals related to derivatives of target position (i.e., target velocity and acceleration) could be used to predict the future position of the target, thus providing a simple predictive mechanism for decreasing time lags. However, models of manual and smooth pursuit tracking typically do not take advantage of this strategy and instead, inputs related to target velocity are used to derive an error signal to drive hand or eye acceleration (Engel and Soechting 2000; Churchland and Lisberger 2001). Thus, these tracking models do not explain the relatively short tracking lags in the present study. Instead, the short lags in our study may be due to the use of an internal model (of a particular shape, or more simply, of a smooth continuation of a 2/3 power trajectory) as the target for the tracking response.

In contrast to the smooth tracking systems, the saccades that typically interrupt smooth pursuit are thought to take advantage of a simple predictive mechanism (using velocity to estimate future position), since they are typically aimed at a future location of a moving target (Engel et al. 1999). In addition, the saccadic eye movements system can also draw upon memory and other internal representations, perhaps to an even greater 
extent than smooth tracking. Eye tracking during the Duncker illusion (1929) illustrates this proposed dichotomy of saccades drawing more heavily on internal representations and smooth pursuit being more simply driven by visual error. When a target moves vertically through a field of horizontally moving dots, it creates a strong illusion of diagonal motion, in the direction opposite to the background motion. Interestingly, both manual interception and the saccadic eye movements exhibit errors in the direction of this illusion. However, the smooth pursuit system keeps the gaze faithfully on target, apparently not drawing upon the illusory representation (Zivotofsky et al. 1996; Soechting et al. 2001).

Tracking in depth

As expected, we found that manual tracking was better in the horizontal and vertical dimensions than in the depth dimension. The positional and directional errors were greater in depth, and the target/hand correlations for both position and velocity were significantly worse in depth. Although our study was not designed to determine the frequency response, we also reported evidence to suggest a meager amplitude gain for depth position, a good response to smooth changes in depth velocity and a poor response to abrupt changes in depth velocity (Fig. 4C and 4D). Thus the time lag for tracking depth velocity was similar to that for the horizontal and vertical dimensions (Fig. 7D), while other aspects of the depth response seemed quite different.

Although it was not clear exactly how frontal plane and depth tracking would differ, differences were expected, mainly due to the differential involvement of conjugate and vergence eye movements, for tracking in the frontal plane and tracking in depth, respectively. As mentioned in the introduction, oculomotor tracking is much better in the 
frontal plane than in depth (Gielen et al. 2004). Furthermore, the vergence eye movements are more variable than smooth pursuit, and the vergence system has complex interactions with the conjugate eye movement systems (Chaturvedi and Gisbergen 1998; Semmlow et al. 1998). Assuming that the eye and hand both followed the target in our task, these complications in oculomotor control were expected to have a counterpart in manual control.

However, in another sense, the poor performance in depth seems somewhat contrary to the well-know ability of humans to estimate time to contact for catching. Stereopsis and time to contact cues (i.e., the rate of retinal expansion) have been shown to strongly contribute to accurate catching performance (Mazyn et al. 2004; Savelsbergh et al. 1992, 1993). Recently, however, Zago and colleagues (2004) have pointed out that pure visual cues are not sufficient to explain subjects' behavior in a manual interception task. Instead, subjects' interception behavior relied heavily upon an internal model of a real ball falling with acceleration due to gravity, or, alternatively, a video image of a ball moving downward with constant speed.

\section{ACKNOWLEDGEMENT}

We thank Professor John F. Soechting for helpful discussions.

\section{GRANTS}

This work was supported by NIH grant R01 NS27484. LA Mrotek was partially supported by an NSF IGERT training grant. 


\section{REFERENCES}

Adamovich SV, Berkinblit MB, Fookson O, and Poizner H. Pointing in 3D space to remembered targets. I. Kinesthetic versus visual target presentation. J Neurophysiol 79: 2833-2846, 1998.

Admiraal MA, Keijsers NLW, and Gielen CCAM. Interaction between gaze and pointing toward remembered visual targets. J Neurophysiol 90: 2136-2148, 2003.

Barnes GR, Barnes DM, and Chakraborti SR. Ocular pursuit responses to repeated, single-cycle sinusoids reveal behavior compatible with predictive pursuit. J Neurophysiol 84:2340-2355, 2000.

Barnes GR and Marsden JF. Anticipatory control of hand and eye movements in humans during oculo-manual tracking. J Physiol 539: 317-330, 2002.

Bock O. Contribution of retinal versus extraretinal signals towards visual localization in goal-directed movements. Exp Brain Res 64: 476-482, 1986.

Churchland MM and Lisberger SG. Experimental and computational analysis of monkey smooth pursuit eye movements. J Neurophysiol 86:741-759, 2001.

Chaturvedi V and Van Gisbergen JA. Shared target selection for combined versionvergence eye movements. J Neurophysiol 80:849-862, 1998.

Dallos PJ and Jones RW. Learning behavior of the eye fixation control system. IEEE Trans Automat Control 8:218-227, 1963.

Desmurget $\mathbf{M}$ and Grafton S. Forward modeling allows feedback control for fast reaching movements. Trends Cogn Sci 4:423-431, 2000.

Duncker K. Uber induzierte Bewegung. Psychol Forsch 12:180-259, 1929.

Engel KC, Anderson JH, and Soechting JF. Oculomotor tracking in two dimensions. $J$ Neurophysiol 81:1597-1602, 1999.

Engel KC and Soechting JF. Manual tracking in two dimensions. $J$ Neurophysiol 83: 3483-3496, 2000.

Flanders M, Mrotek LA, and Gielen CCAM. Planning and drawing complex shapes. $J$ Neurophysiol (submitted).

Gielen CCAM, Gabel SF, and Duysens J Retinal slip during active head motion and stimulus motion. Exp Brain Res 155: 211-219.2004.

Henriques DYP, Klier EM, Smith MA, Lowy D, and Crawford JD. Gaze-centered remapping of remembered visual space in an open-loop pointing task. J Neurosci 18: 1583-1594, 1998.

Kettner RE, Leung HC, and Peterson BW. Predictive smooth pursuit of complex twodimensional trajectories in monkey: component interactions. Exp Brain Res 108: 221235, 1996. 
Krakauer JW, Ghilardi, M-F, and Ghez C. Independent learning of internal models for kinematic and dynamic control of reaching. Nature Neurosci 2:1026-1031, 1999.

Lacquaniti F, Terzuolo C, and Viviani P. The law relating the kinematic and figural aspects of drawing movements. Acta Psychol Amst 54: 115-130, 1983.

Mazyn LI, Lenoir M, Montagne G, and Savelsbergh GJ. The contribution of stereo vision to one-handed catching. Exp Brain Res 157: 383-390, 2004.

Medendorp WP and Crawford JD. Visuospatial updating of reaching targets in near and far space. Neuroreport 13: 633-636, 2002.

Poulton EC. Tracking Skill and Manual Control. Academic Press, London. 1974.

Prablanc C and Martin O. Automatic control during hand reaching at undetected twodimensional target displacements. J Neurophysiol 67: 455-469, 1992.

Roitman AV, Massaquoi SG, Takahashi K, and Ebner TJ. Kinematic analysis of manual tracking in monkeys: characterization of movement intermittencies during a circular tracking task. J Neurophysiol 91: 901-911, 2004.

Savelsbergh GJ, Whiting HT, Burden AM, and Bartlett RM. The role of predictive visual temporal information in the coordination of muscle activity in catching. Exp Brain Res 89: 223-228, 1992.

Savelsbergh GJ, Whiting HT, Pijpers JR, and van Santvoord AA. The visual guidance of catching. Exp Brain Res 93: 148-156, 1993.

Semmlow JL, Yuan W, Alvarez TL. Evidence for separate control of slow version and vergence eye movements: support for Hering's Law. Vision Res 38:1145-1152, 1998.

Smith WM and Bowen KF. The effects of delayed and displaced visual feedback on motor control. J Mot Behav 12: 91-101, 1980.

Soechting JF and Flanders $\mathbf{M}$. Sensorimotor representations for pointing to targets in three-dimensional space. J Neurophysiol 62: 582-594, 1989a.

Soechting JF and Flanders $\mathbf{M}$. Errors in pointing are due to approximations in sensorimotor transformations. J Neurophysiol 62: 595-608, 1989 b.

Soechting JF, Lacquaniti F, and Terzuolo CA. Coordination of arm movements in three-dimensional space. Sensorimotor mapping during drawing movement.

Neuroscience 17: 295-311, 1986.

Soechting JF, Engel KC, and Flanders M. The Duncker illusion and eye-hand coordination. J Neurophysiol 85:843-854, 2001.

Soechting JF and Terzuolo CA. An algorithm for the generation of curvilinear wrist motion in an arbitrary plane in three-dimensional space. Neurosci 19: 1393-1405, 1986.

Soechting JF and Terzuolo CA. Organization of arm movements. Motion is segmented. Neurosci 23: 39-51, 1987a.

Soechting JF and Terzuolo CA. Organization of arm movements in three-dimensional space. Wrist motion is piecewise planar. Neurosci 23: 53-61, $1987 \mathrm{~b}$. 
Viviani P, Campadelli P, and Mounoud P. Visuo-manual pursuit tracking of human two-dimensional movements. J Exp Psychol Hum Percept Perform 13: 62-78, 1987.

Wolpert DM, Ghahramani Z, and Jordan MI. An internal model for sensorimotor integration. Science 269:1880-1882, 1995.

Yasui S and Young LR. Percieved visual motion as an effective stimulus to pursuit eye movement system. Science 190:906-908, 1975.

Zago M, Bosco G, Maffei V, Iosa M, Ivanenko YP, Lacquaniti F. Internal models of target motion: expected dynamics overrides measured kinematics in timing manual interceptions. J Neurophysiol 91:1620-1634, 2004.

Zelaznik HZ, Hawkins B, and Kisselburgh L. Rapid visual feedback processing in single-aiming movements. J Mot Behav 15: 217-236, 1983.

Zivotofsky AZ, Rottach KG, Averbuch-Heller L, Kori AA, Thomas CW, Dell'Osso LF, and Leigh RJ. Saccades to remembered targets: the effects of smooth pursuit and illusory stimulus motion. J Neurophysiol 76:3617-3632, 1996. 
Table 1 Shape Properties

Cassini ellipse shapes

Orientations

Front, Side

Bend Locations

Long axis, Short axis, Oblique axis

Bend Magnitude $\quad 30^{\circ}, 60^{\circ}$

\begin{tabular}{c|l}
\hline \hline 4-Plane shapes & \\
\hline Size & Small, Medium, Large \\
\hline
\end{tabular}

Table 2 Main effects of cycle for each dependent variable (All Shapes)

\begin{tabular}{l|cccc}
\hline \multicolumn{1}{c}{$F$} & $\mathrm{df}$ & $p$ & post hoc \\
\hline Distance Error & 2.575 & 3,404 & 0.054 & \\
Abs. Speed Error & 12.941 & 3,404 & $<0.001$ & $\mathbf{1}>2,3,4$ \\
FP Direction Error & 0.188 & 3,404 & 0.905 & \\
SP Direction Error & 3.529 & 3,404 & 0.015 & $\mathbf{1}>3$ \\
& & & & \\
X Position Lag & 44.323 & 3,404 & $<0.001$ & $\mathbf{1}>2,3,4$ \\
Y Position Lag & 4.132 & 3,325 & 0.007 & $\mathbf{1}>4$ \\
Z Position Lag & 15.440 & 3,403 & $<0.001$ & $\mathbf{1}>2,3,4$ \\
& & & & \\
X Velocity Lag & 65.628 & 3,403 & $<0.001$ & $\mathbf{1}>2,3,4$ \\
Y Velocity Lag & 11.865 & 3,325 & $<0.001$ & $\mathbf{1}>2,3,4$ \\
Z Velocity Lag & 14.656 & 3,403 & $<0.001$ & $\mathbf{1}>2,3,4$ \\
& & & & \\
FP Direction Lag & 3.298 & 3,403 & 0.020 & $\mathbf{1}>4$ \\
SP Direction Lag & 6.954 & 3,392 & $<0.001$ & $\mathbf{1}>2,3,4$
\end{tabular}


Table 3 ANOVA's comparing bend types (Cassini shapes only)

\begin{tabular}{|c|c|c|c|}
\hline & $F_{(3,332)}$ & $p$ & post hoc \\
\hline $\begin{array}{l}\text { Section } 1 \\
\text { distance error } \\
\text { speed error } \\
\text { FP direction error } \\
\text { SP direction error } \\
\text { instantaneous lag } \\
\end{array}$ & $\begin{array}{c}0.857 \\
20.091 \\
19.708 \\
19.246 \\
3.652 \\
\end{array}$ & $\begin{array}{c}0.464 \\
<0.001 \\
<0.001 \\
<0.001 \\
0.013 \\
\end{array}$ & $\begin{array}{l}\text { Long }>\text { All } \\
\text { Long }>\text { All } \\
\text { Long }>\text { All } \\
\text { Long }>\text { Oblique } \\
\end{array}$ \\
\hline $\begin{array}{l}\text { Section } 2 \\
\text { distance error } \\
\text { speed error } \\
\text { FP direction error } \\
\text { SP direction error } \\
\text { instantaneous lag }\end{array}$ & $\begin{array}{c}1.397 \\
2.471 \\
13.230 \\
18.634 \\
4.465 \\
\end{array}$ & $\begin{array}{c}0.244 \\
0.062 \\
<0.001 \\
<0.001 \\
0.004\end{array}$ & $\begin{array}{l}\text { Long, Short }>\text { No, Oblique } \\
\text { Short }>\text { All } \\
\text { Short }>\text { Oblique }\end{array}$ \\
\hline $\begin{array}{l}\text { Section } 3 \\
\text { distance error } \\
\text { speed error } \\
\text { FP direction error } \\
\text { SP direction error } \\
\text { instantaneous lag } \\
\end{array}$ & $\begin{array}{c}1.968 \\
8.779 \\
67.631 \\
13.714 \\
4.501 \\
\end{array}$ & $\begin{array}{c}0.002 \\
<0.001 \\
<0.001 \\
<0.001 \\
0.004 \\
\end{array}$ & $\begin{array}{l}\text { Oblique }>\text { No, Long } \\
\text { Oblique }>\text { Long, Short } \\
\text { Oblique }>\text { All; No }>\text { Short } \\
\text { Oblique }>\text { All } \\
\text { Short }>\text { Oblique } \\
\end{array}$ \\
\hline $\begin{array}{l}\text { Section } 4 \\
\text { distance error } \\
\text { speed error } \\
\text { FP direction error } \\
\text { SP direction error } \\
\text { instantaneous lag }\end{array}$ & $\begin{array}{c}4.963 \\
33.113 \\
10.291 \\
15.863 \\
3.938 \\
\end{array}$ & $\begin{array}{c}0.002 \\
<0.001 \\
<0.001 \\
<0.001 \\
0.009\end{array}$ & $\begin{array}{l}\text { Oblique }>\text { Short } \\
\text { Long }>\text { All } \\
\text { Long }>\text { No, Oblique } \\
\text { Long }>\text { Oblique }>\text { No; Short }>\text { No } \\
\text { Long, Oblique }>\text { No }\end{array}$ \\
\hline $\begin{array}{l}\text { Section } 5 \\
\text { distance error } \\
\text { speed error } \\
\text { FP direction error } \\
\text { SP direction error } \\
\text { instantaneous lag }\end{array}$ & $\begin{array}{c}2.605 \\
7.892 \\
1.269 \\
26.851 \\
5.808 \\
\end{array}$ & $\begin{array}{c}0.052 \\
<0.001 \\
0.285 \\
<0.001 \\
<0.001\end{array}$ & $\begin{array}{l}\text { Short }>\text { No, Long } \\
\text { Short }>\text { All; Oblique }>\text { Long } \\
\text { Oblique }>\text { No, Short }\end{array}$ \\
\hline $\begin{array}{l}\text { Section } 6 \\
\text { distance error } \\
\text { speed error } \\
\text { FP direction error } \\
\text { SP direction error } \\
\text { instantaneous lag }\end{array}$ & $\begin{array}{c}3.949 \\
22.291 \\
51.770 \\
19.511 \\
6.217\end{array}$ & $\begin{array}{l}0.009 \\
<0.001 \\
<0.001 \\
<0.001 \\
<0.001\end{array}$ & $\begin{array}{l}\text { Oblique }>\text { No } \\
\text { Oblique }>\text { Long }>\text { No, Short } \\
\text { Oblique }>\text { All } \\
\text { Oblique }>\text { All } \\
\text { Oblique }>\text { Long, No }\end{array}$ \\
\hline
\end{tabular}


Table 4 Strength of hand-target correlations for Cassini Shapes (all cycles)

\begin{tabular}{l|cc|ccc}
\hline & Shifted r & Shifted Z-score & $F^{\#}$ & $\mathrm{df}$ & $p$ \\
\hline X position & $0.993 \pm 0.004$ & $0.393 \pm 0.041$ & & & \\
Y position* & $0.849 \pm 0.156$ & $-0.979 \pm 1.477$ & 286.004 & 2,935 & $<0.001$ \\
Z position & $0.992 \pm 0.010$ & $0.382 \pm 0.093$ & & & \\
& & & & & \\
X velocity & $0.937 \pm 0.034$ & $0.579 \pm 0.190$ & & & \\
Y velocity* & $0.628 \pm 0.190$ & $-1.143 \pm 1.057$ & 784.402 & 2,984 & $<0.001$ \\
Z velocity & $0.922 \pm 0.041$ & $0.493 \pm 0.227$ & & & \\
& & & & & \\
\hline
\end{tabular}

* different than others

" ANOVA's were performed on Z-scores

Table 5 Strength of hand-target correlations for 4-Plane Shapes (all cycles)

\begin{tabular}{l|cc|ccc}
\hline & Shifted r & Shifted Z-score & $F^{\#}$ & $\mathrm{df}$ & $p$ \\
\hline X position & $0.996 \pm 0.002$ & $0.430 \pm 0.018$ & & & \\
Y position* & $0.825 \pm 0.171$ & $-0.961 \pm 1.391$ & 70.657 & 2,202 & $<0.001$ \\
Z position & $0.995 \pm 0.005$ & $0.423 \pm 0.039$ & & & \\
& & & & & \\
X velocity & $0.943 \pm 0.030$ & $0.439 \pm 0.196$ & & & \\
Y velocity* & $0.739 \pm 0.208$ & $-0.918 \pm 1.375$ & 62.769 & 2,203 & $<0.001$ \\
Z velocity & $0.936 \pm 0.040$ & $0.389 \pm 0.262$ & & & \\
& & & & & \\
\hline * different than others
\end{tabular}




\section{Table Legends}

Table 1 Seventeen different shapes were presented. Cassini shapes were presented in front and side view. Each of the Cassini shapes could have no bend or could be bent along a long, short or oblique axis by 30 or $60 \mathrm{deg}$. The 4-plane shape was presented in small, medium and large sizes.

Table 2 Statistical data to illustrate that the first cycle (bold “1”) revealed significantly larger errors than the other cycles for most measures.

Table 3 Statistical analysis of errors for position, speed, direction in frontal plane (FP) and sagittal plane (SP), and lag for the six sections of the Cassini shape between the bends (see Fig. 1A). The hypothesis was tested that the section of the cycle after a bend would have more error than the corresponding section where there was no bend. The fourth column shows the results of a post-hoc analysis. In each case, the type of shape predicted to have more error is listed in boldface type.

Table 4 Correlation values between target (Cassini shape) and hand for position and velocity. Correlation values for the $Y$-dimension (depth) were significantly lower than for the other dimensions.

Table 5 Correlation values between target (4-Plane shape) and hand for position and velocity. Correlation values for the $Y$-dimension (depth) were significantly lower than for the other dimensions. 


\section{Figure Legends}

Figure 1 Schematic of Cassini shape, showing the axes along which bends occured (A). The six sections are numbered; their boundaries are defined by each possible bend location. One 4-Plane shape is shown (B). This shape was made by joining the ends of four semi-circles. For clarity, this shape is shown rotated from the subject's perspective ( $20^{\circ}$ around the vertical axis and $30^{\circ}$ around the horizontal axis).

Figure 2 The main shape used in this experiment was a peanut-shaped Cassini ellipse (A). It was in a plane rotated $45^{\circ}$ about the horizontal $(X)$ axis, such that the bottom of the shape was closer to the subject. To generate another orientation (B) we rotated the shape $80^{\circ}$ about the vertical $(Y)$ axis, so that subjects saw a side view. In addition to these shapes, we altered the Cassini ellipse by folding it along three axes. It was bent along the long axis ( $C$ and $D)$, the short axis ( $E$ and $F$ ) and an oblique axis ( $G$ and $H)$. In this Figure, the magnitude of all the bends is $60^{\circ}$, but we also bent each shape $30^{\circ}$. For all conditions, the subject began at the location of the discontinuity and moved clockwise around the shape. All the shapes are shown from the perspective of the subject.

Figure 3 The target path for four shapes is shown with the thick dashed line: Front No Bend shape (A), Medium 4-Plane shape (B), Side No Bend shape (C), Side $60^{\circ}$ Long Axis Bend shape (D). One subject's tracking performance is also shown. The data from the first cycle are graphed as a thick line and cycles 2-4 are graphed as thin lines. Arrows denote the starting point and direction.

Figure 4 Patterns of target and subject motion for the Side $60^{\circ}$ Long Axis Bend shape. This is the same trial as presented in Fig. 3D. In each graph, the data from the first cycle are graphed as a thick line and cycles 2-4 are graphed as thin lines. The target motion is graphed with thick dashed black lines. Vertical double lines indicate times when the target was at an indent of the shape. The vertical single lines (including times 0 and $5000 \mathrm{~ms}$ ) indicate times when this shape had an abrupt bend. The top, left panel 
shows the frontal plane, horizontal position (A); the middle, left panel shows the position in depth (C); and the bottom, left panel shows the vertical position (E). The top, right panel shows the horizontal velocity (B); the middle, right panel shows the velocity in depth (D); and the bottom, right panel shows the vertical velocity (F).

Figure 5 Direction was calculated in two planes: the frontal plane (A) and the sagittal plane (B). The direction of the target and the hand are plotted over time for the same trial as in Fig. 3D and Fig. 4 (Side 60 ${ }^{\circ}$ Long Axis Bend). Target direction is shown with the thick, dashed black lines. Vertical double lines indicate times when the target was at an indent of the shape and vertical single lines show the times when the target was at an abrupt bend. Hand direction from the first cycle is graphed as a thick line, and cycles 2-4 are graphed as thin lines.

Figure 6 Average distance (A) and speed (B-C) errors across time for all Cassini shapes (all subjects). The error for the first cycle is graphed with thick red lines and cycles 2-4 are graphed with thin blue lines. The vertical hatching around each line represents $95 \%$ confidence intervals. The vertical double lines indicate times when the target reached the indents of the shape and the single vertical lines indicate the time when the target abruptly changed direction (all possible bends).

Figure 7 The average error and lag values (all subjects and shapes) are plotted for each cycle (error bars represent \pm 1 standard error of the grand mean for the six subjects). Errors for distance (A), speed (C) and direction (E) are shown in the left column. The lead/lag values for position (B), velocity (D) and direction (F) are shown in the right column. Negative values indicate that the subject was lagging behind the target. Stars were used as the symbol when the first cycle was significantly different from all other cycles. Diamonds were used when the first cycle was different from the fourth cycle and the square indicates that the first cycle was different from the third cycle. The $2^{\text {nd }}, 3^{\text {rd }}$ and $4^{\text {th }}$ cycles were never significantly different from one another. 
Figure 8 The schematic in (A) shows how instantaneous lag was calculated. The current hand position is labeled $\mathrm{H}_{\mathrm{t}}$ and the current target position is labeled $\mathrm{T}_{\mathrm{t}}$. Distance was calculated between the current hand position and the target at all positions (before and including the current position). The pair that produced the least error was found, and the time difference between the pair was computed. This time difference is defined as the instantaneous lag. For the example shown, the lag is $-80 \mathrm{~ms}$. The instantaneous lags for four shapes are shown in this figure (B, C, D, E), with increasing negative (lag) values plotted upwards. The vertical double lines show the time when the target was at the indents. The single vertical lines in (C) show the times when the target finished each semi-circle and changed from horizontal to vertical planes. The lag for the first cycle is graphed with the thick line; the lags for cycles 2-4 are plotted with thin lines. We calculated the average instantaneous lag for all the Cassini shapes and compared the lag during the first cycle with the lag during the other three cycles $(F)$. The first cycle is graphed with a thick red line, the average of all the other cycles with the thin blue line. The vertical hatching around each line represents the 95\% confidence intervals. The vertical double lines show the times when the target was at the indent and the single vertical lines show the times of every possible bend.

Figure 9 For each bend (long axis (A), short axis (B) and oblique axis (C)), we examined the magnitude of the error or lag during each section (sub-cycle) of the Cassini shapes. A section is defined as the part of a shape between possible bends; every shape has six sections (see Fig. 1A). The highlighted areas in the schematics show the sections where we would expect more error and lag, if the bends were problematic. We compared the magnitude of the distance error (dis err), and speed error (second line from inside, spd err), frontal plane direction error (FP err), sagittal plane direction error (second line from outside, SP err) and instantaneous lag (ilag) during each section after each type of bend. The thick dashed lines indicate the sections where there was more error or a longer lag for each bend orientation (see Table 3 for details). 
Figure 1

A Cassini Shapes: All Bend Axes

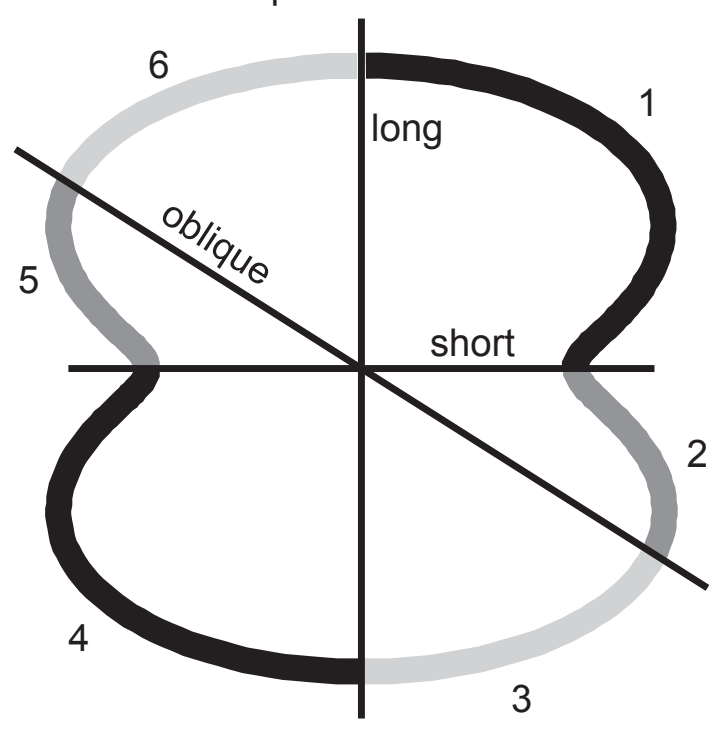

B 4-Plane Shape

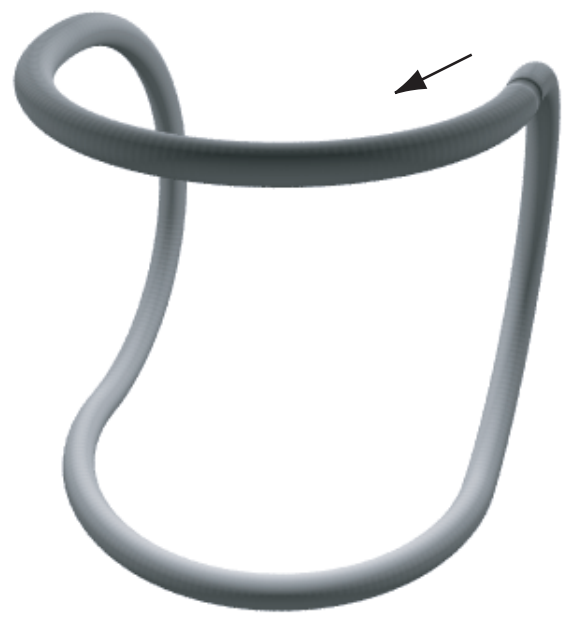


Figure 2

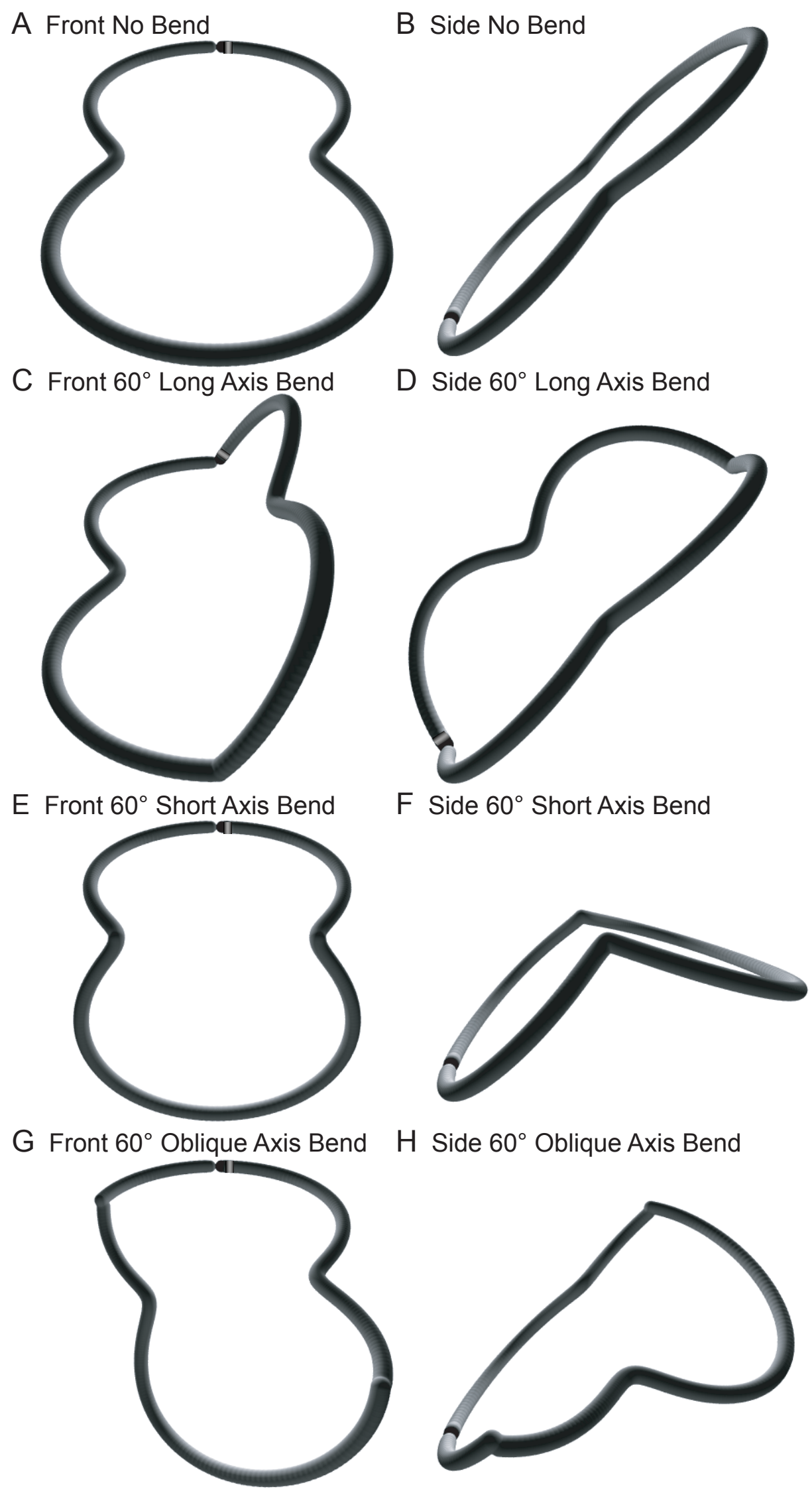


Figure 3

A Front No Bend

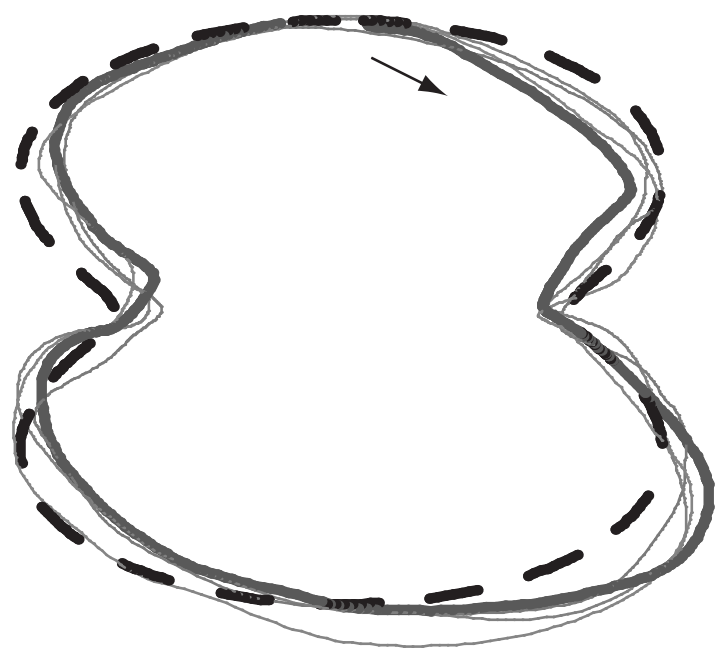

C Side No Bend

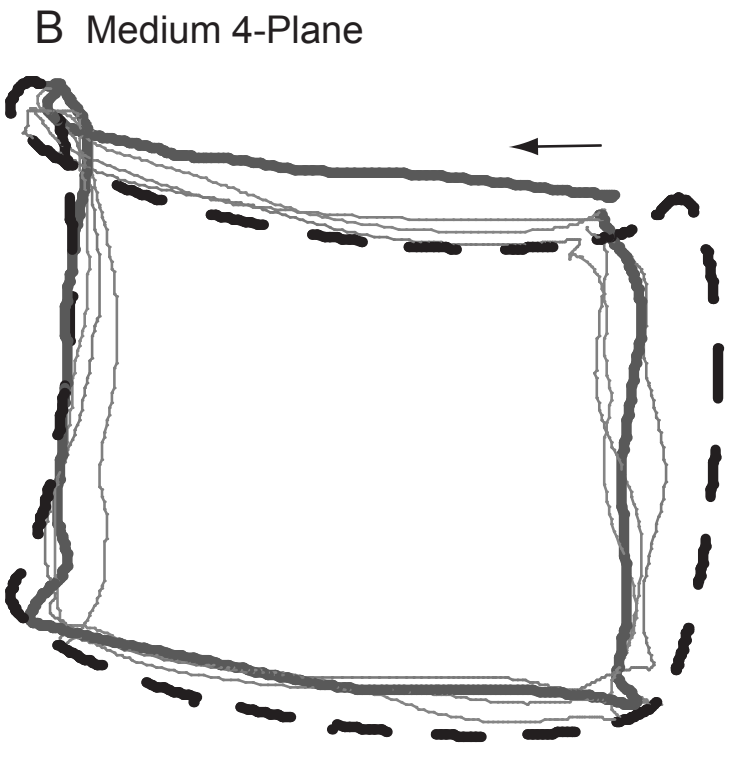

D Side $60^{\circ}$ Long Axis Bend

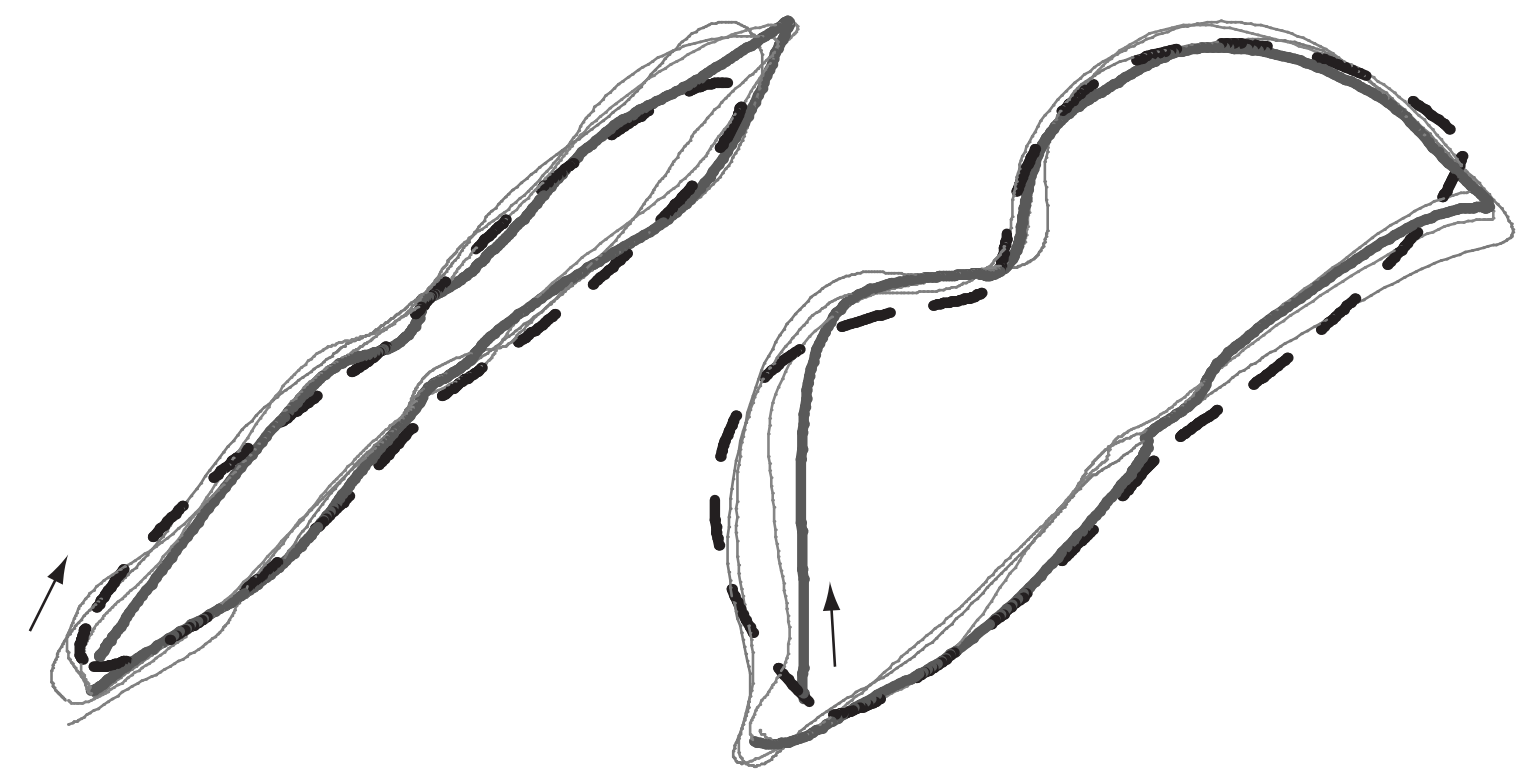


Figure 4

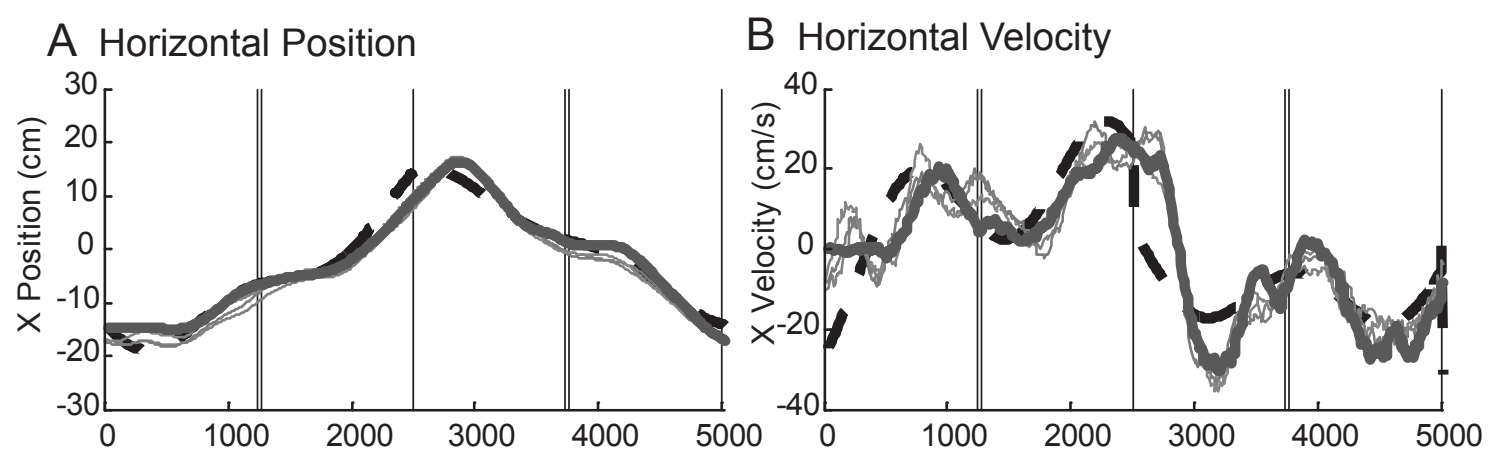

C Depth Position

D Depth Velocity
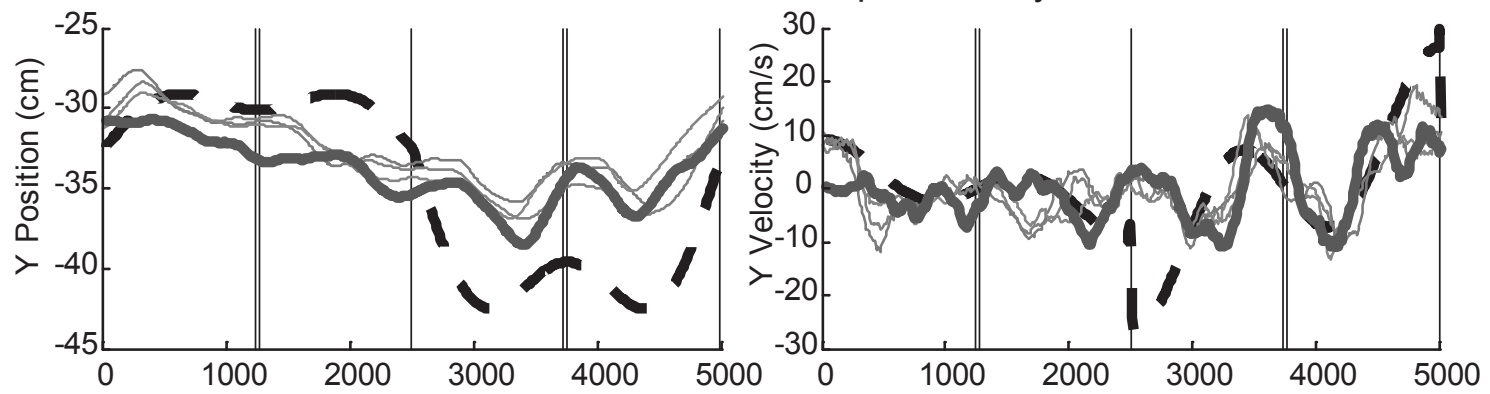

E Vertical Position

F Vertical Velocity
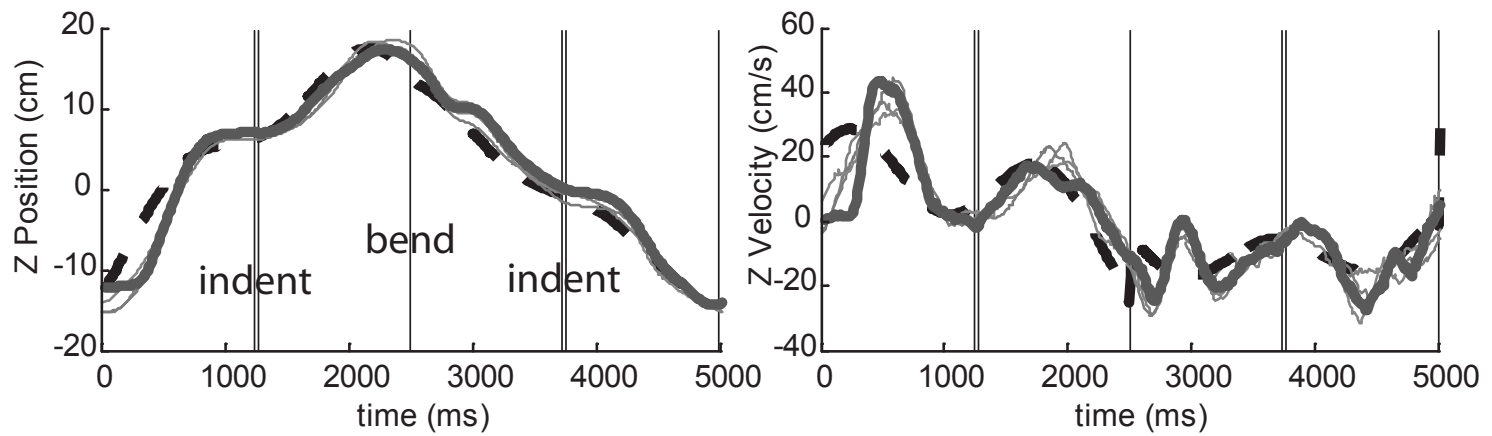
Figure 5

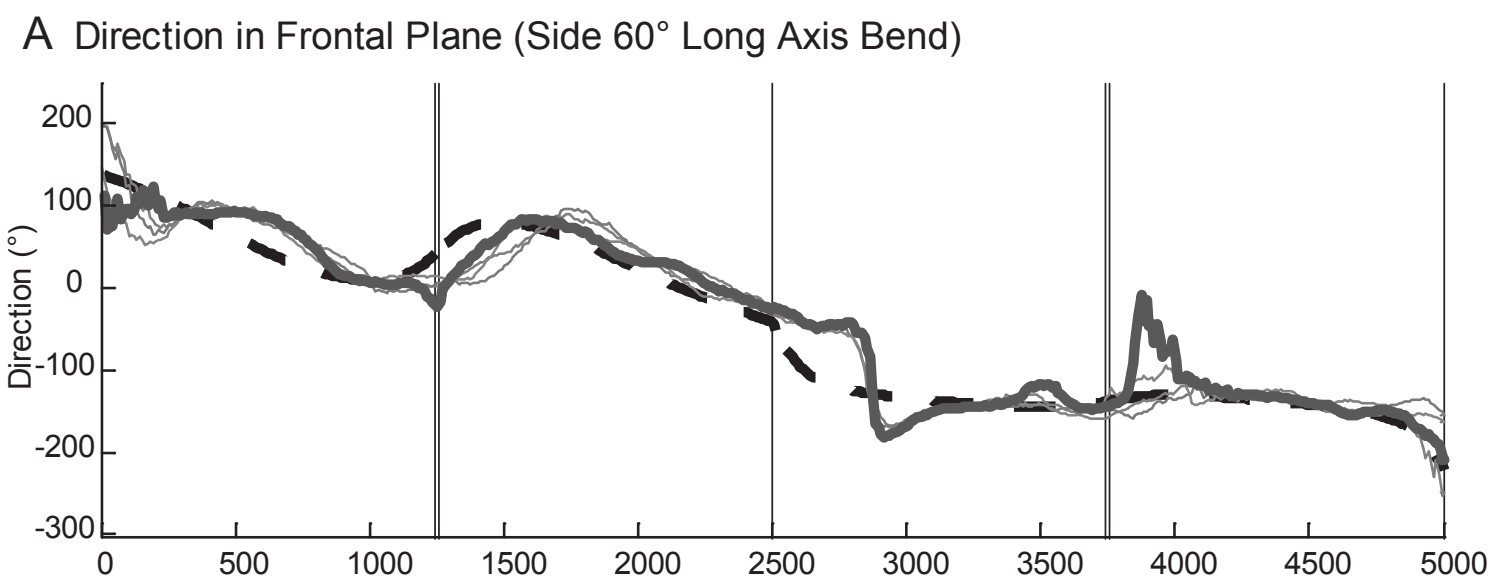

B Direction in Sagittal Plane (Side $60^{\circ}$ Long Axis Bend)

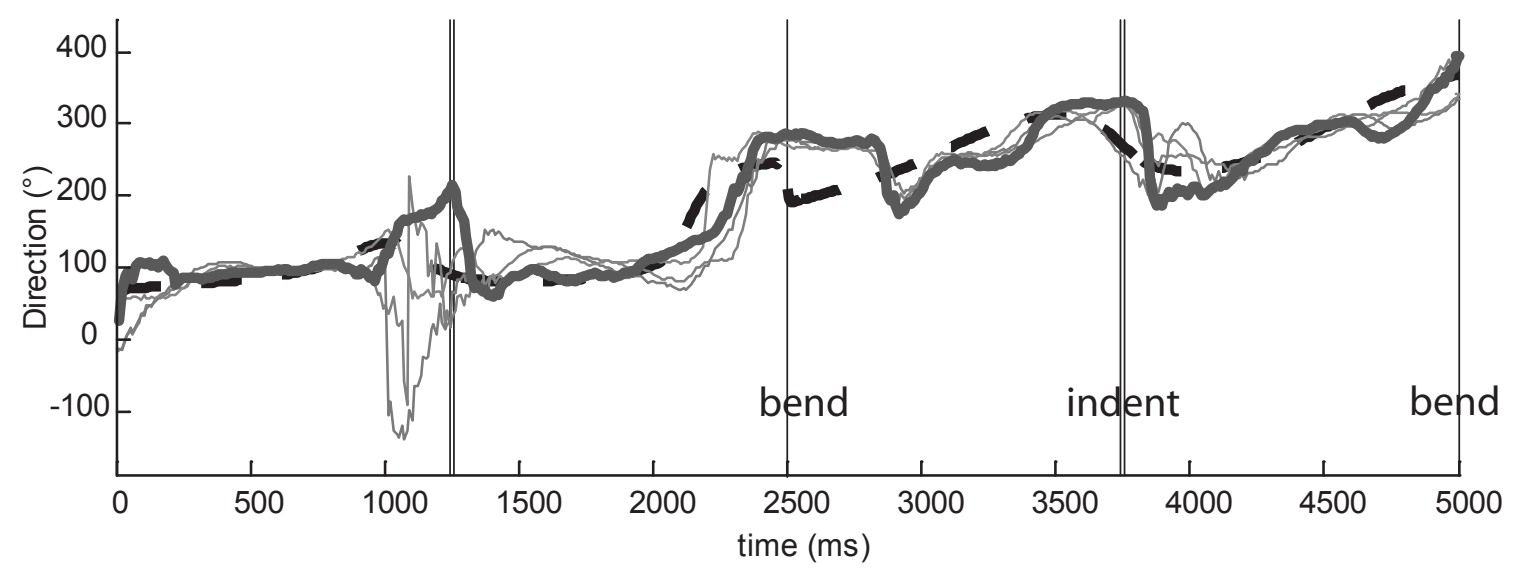


Figure 6

A Distance Error (All Cassini Shapes)

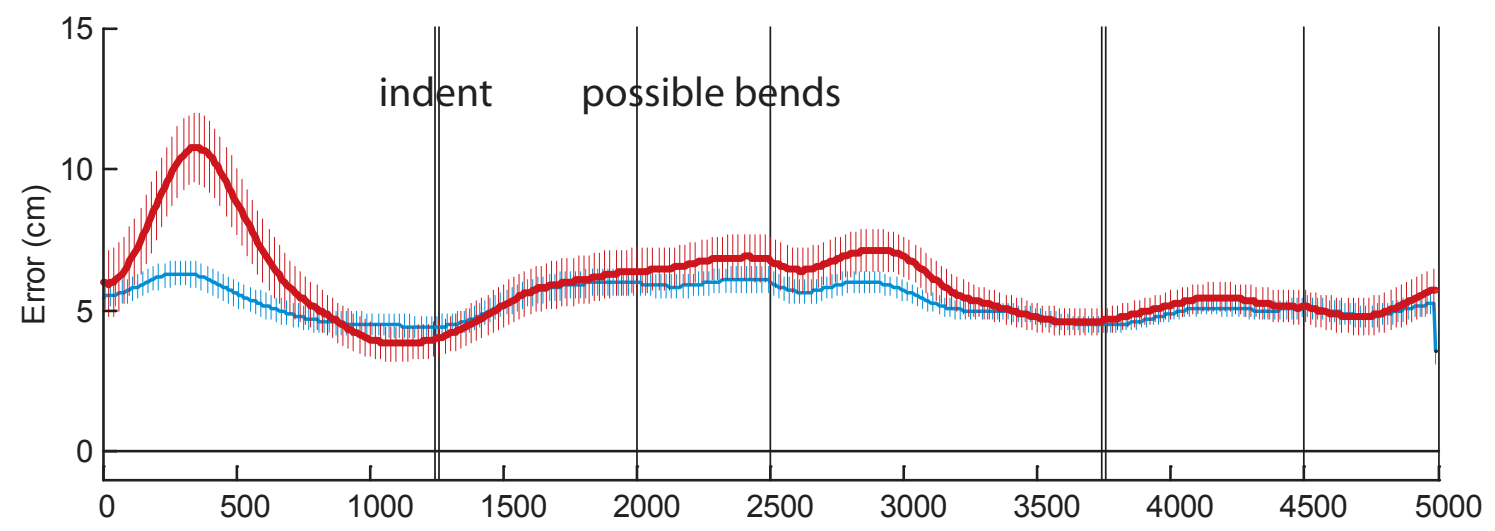

B Unsigned Speed Error (All Cassini Shapes)

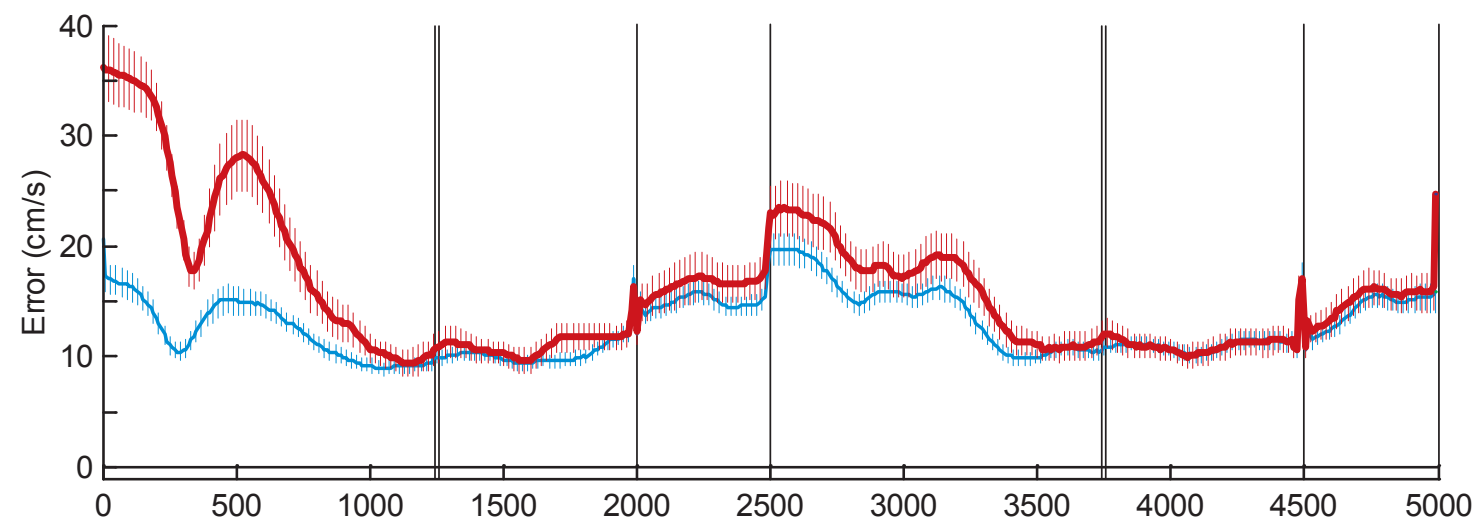

C Signed Speed Error (All Cassini Shapes)

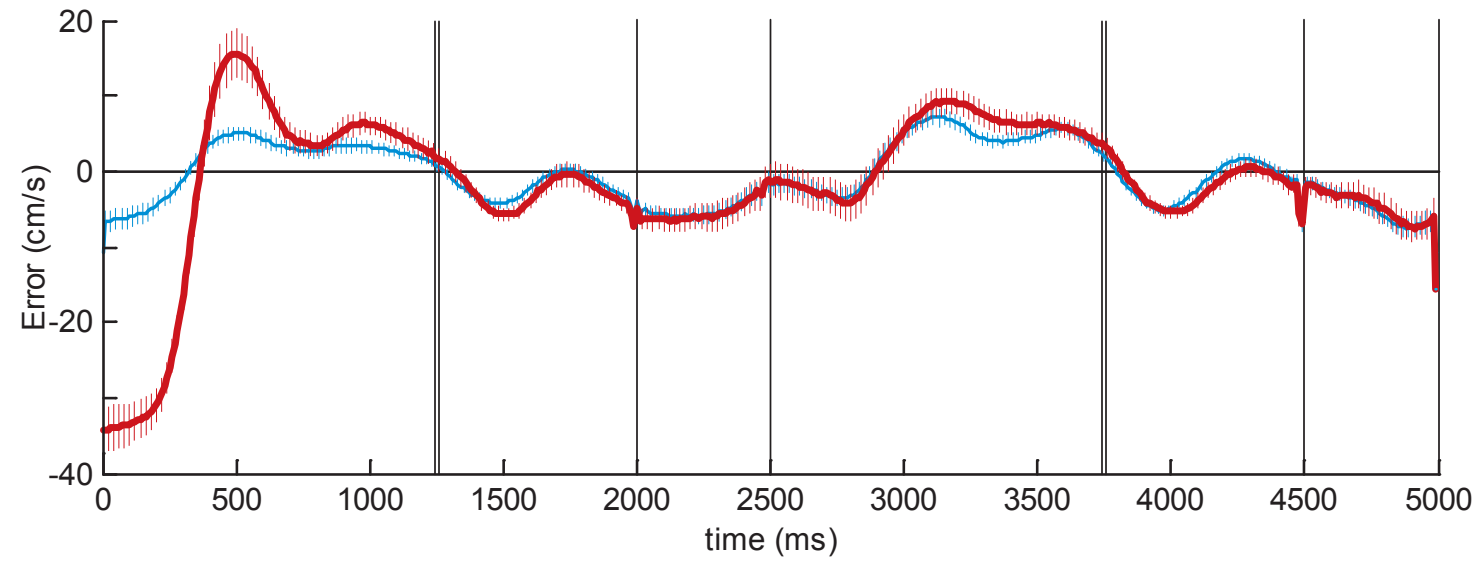


Figure 7
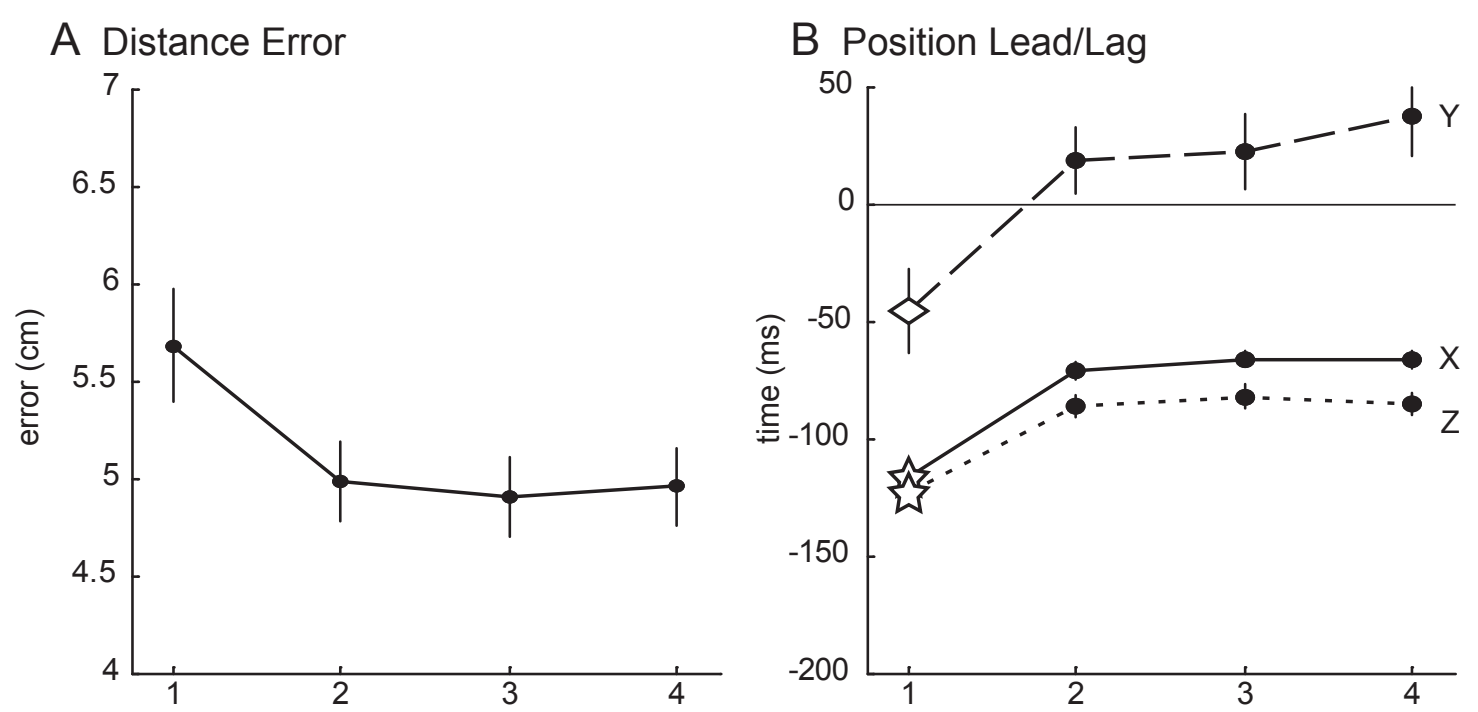

\section{Speed Error}

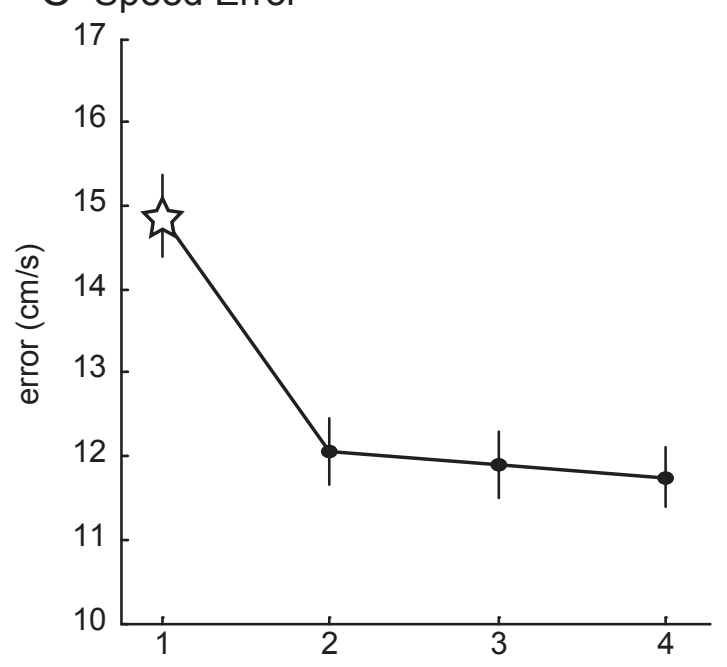

D Velocity Lead/Lag

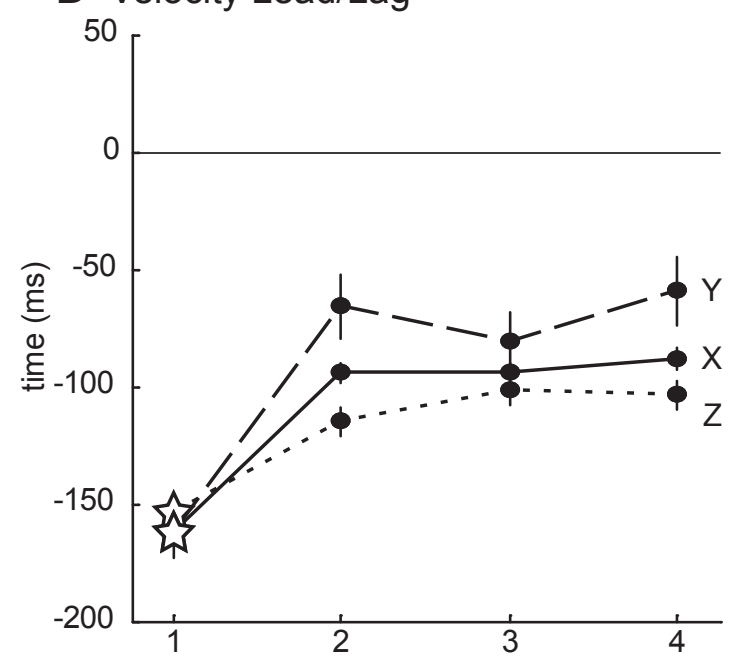

E Absolute Direction Error

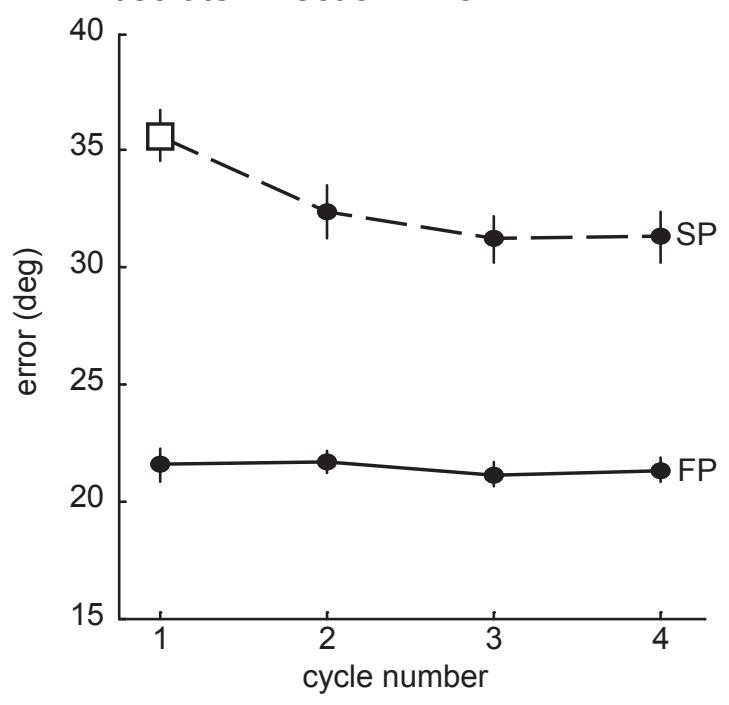

F Direction Lead/Lag

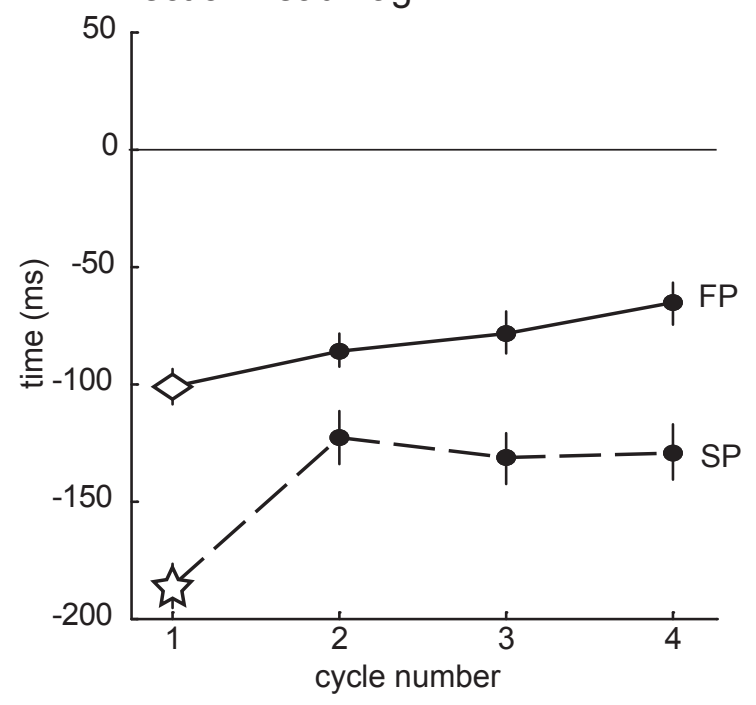


Figure 8

A Instantaneous Lag Calculation Schematic

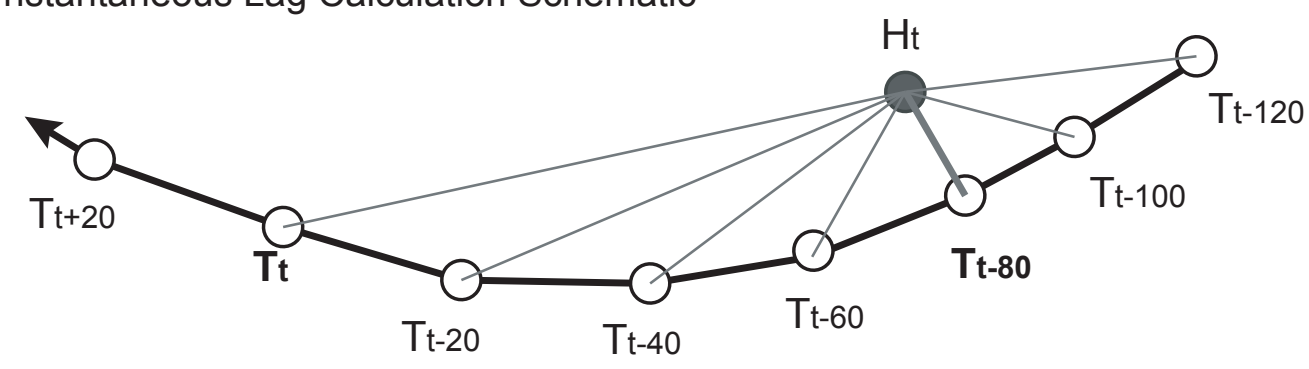

B Front No Bend

C Medium 4-Plane
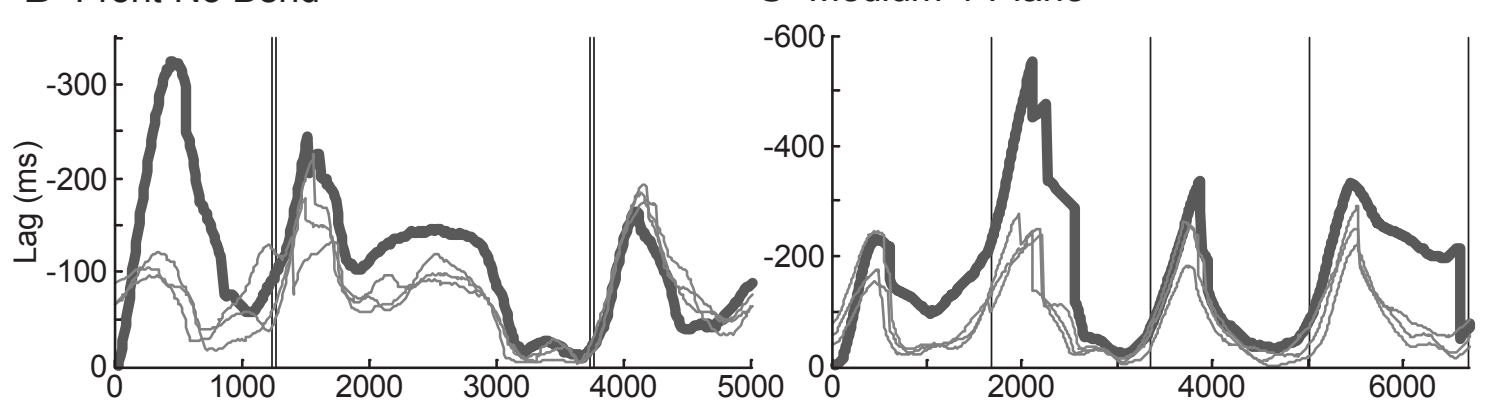

D Side No Bend

E Side $60^{\circ}$ Long Axis Bend

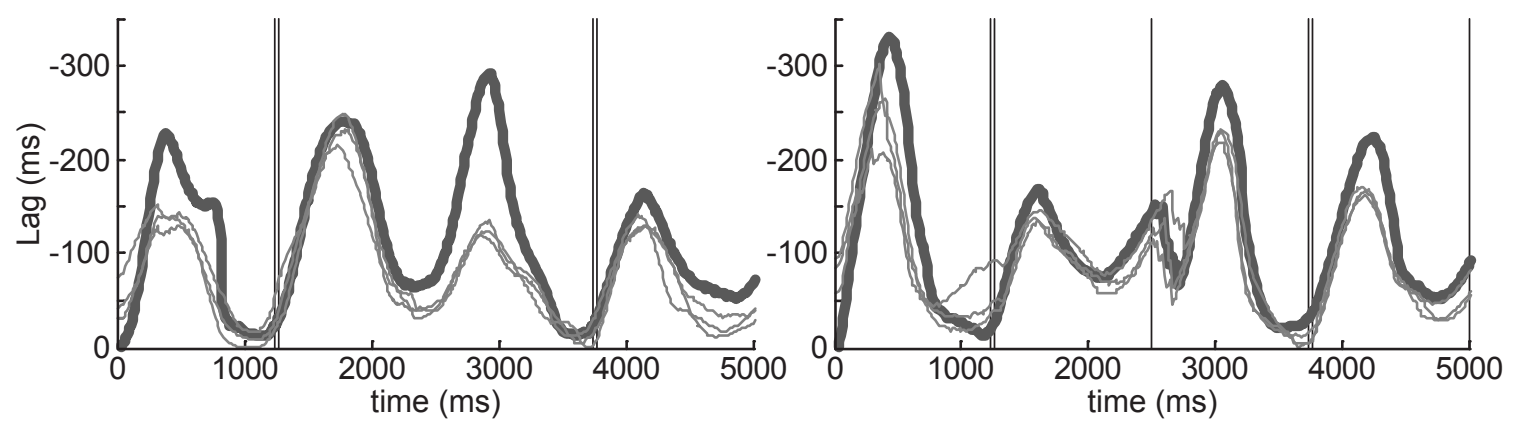

F Instantaneous Lag Average for All Cassini Shapes

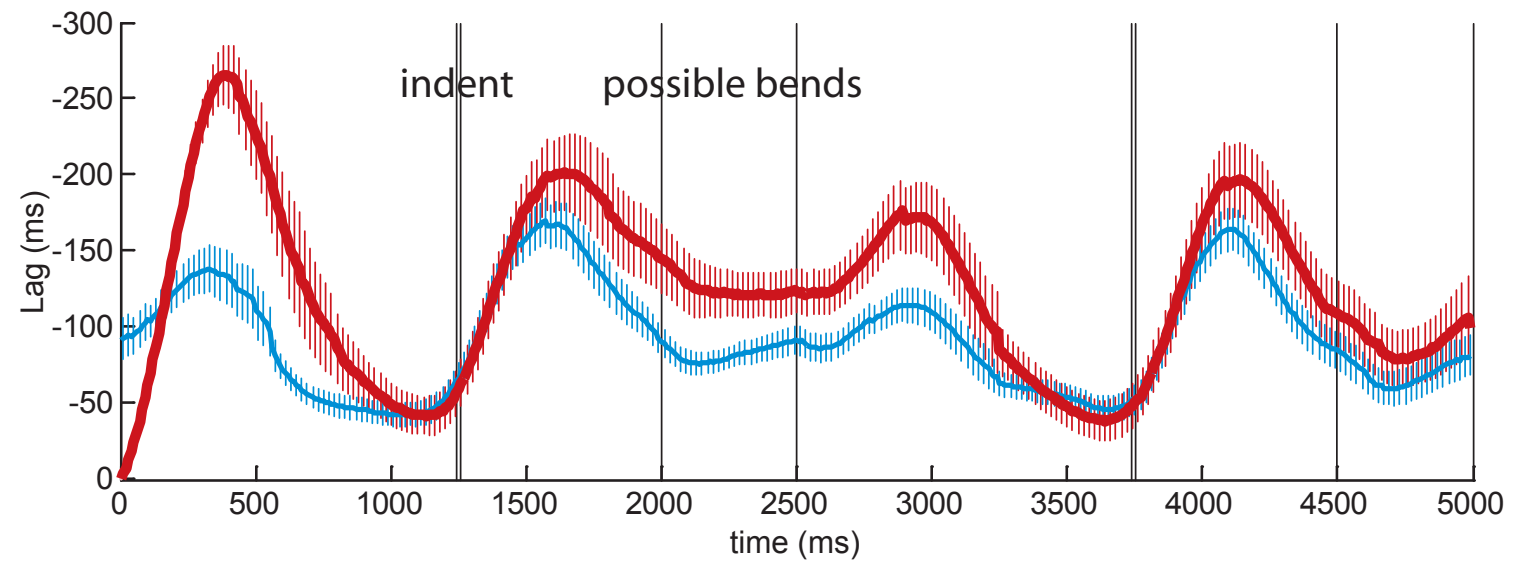


Figure 9

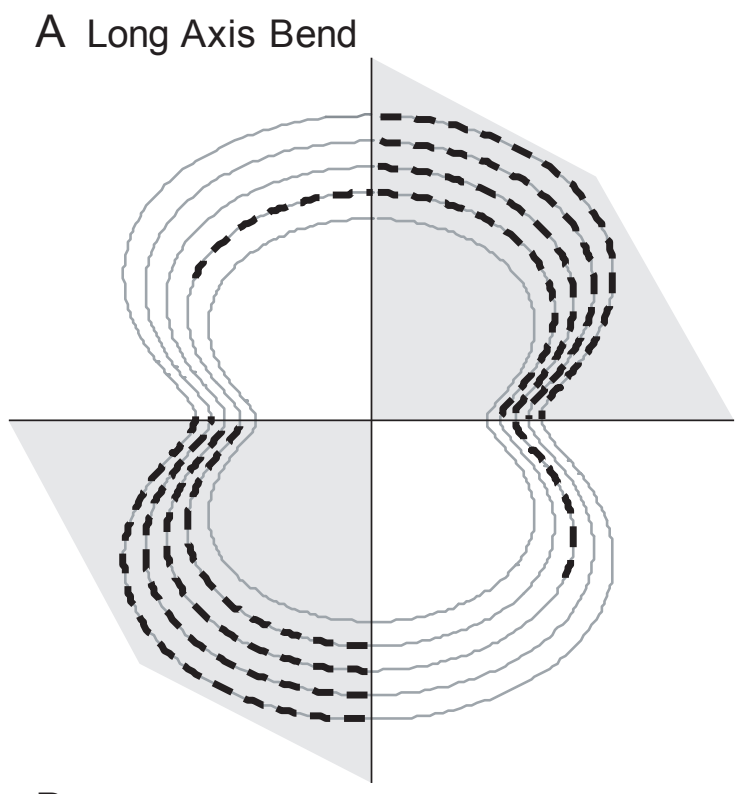

B Short Axis Bend

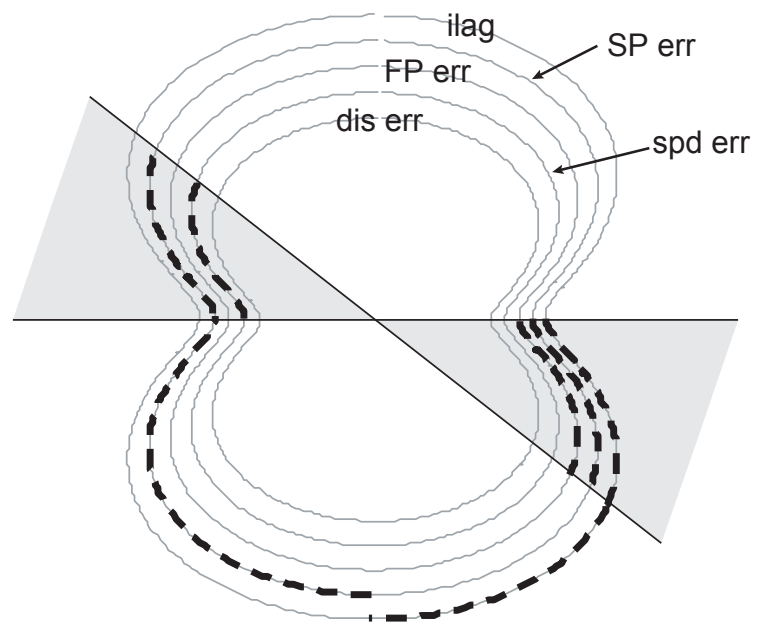

C Oblique Axis Bend

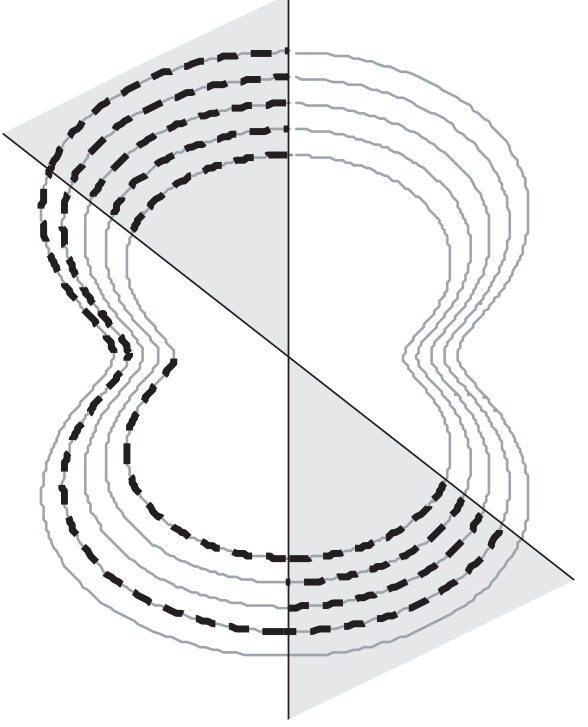

